\title{
Two delays induce Hopf bifurcation and double Hopf bifurcation in a diffusive Leslie-Gower predator-prey system
}

\author{
Yanfei $\mathrm{Du}^{1,2}$, Ben $\mathrm{Niu}^{2}$, and Junjie $\mathrm{Wei}^{2}$ \\ ${ }^{1}$ College of Arts and Sciences, Shaanxi University of \\ Science and Technology, Xi'an 710021, China. and \\ ${ }^{2}$ Department of Mathematics, Harbin Institute of \\ Technology at Weihai, Weihai 264209, China.
}

(Dated: December 10, 2018)

\begin{abstract}
In this paper, the dynamics of a modified Leslie-Gower predator-prey system with two delays and diffusion is considered. By calculating stability switching curves, the stability of positive equilibrium and the existence of Hopf bifurcation and double Hopf bifurcation are investigated on the parametric plane of two delays. Taking two time delays as bifurcation parameters, the normal form on the center manifold near the double Hopf bifurcation point is derived, and the unfoldings near the critical points are given. Finally, we obtain the complex dynamics near the double Hopf bifurcation point, including the existence of quasi-periodic solutions on a 2-torus, quasi-periodic solutions on a 3 -torus, and strange attractors.
\end{abstract}

Keywords: Leslie-Gower model, two delays, double Hopf bifurcation, normal form, strange attractor

* niu@hit.edu.cn; author to whom correspondence should be addressed. 
Diffusive predator-prey models with delays have been investigated widely, and the delay induced Hopf bifurcation analysis has been well studied. However, the study about bifurcation analysis of predator-prey models with two simultaneously varying delays has not been well established. Neither the Hopf bifurcation theorem with two parameters nor the derivation process of normal form for two delays induced double Hopf bifurcation has been proposed in literatures. In this paper, we investigate a diffusive Leslie-Gower model with two delays, and carry out Hopf and double Hopf bifurcation analysis of the model. Applying the method of studying characteristic equation with two delays, we get the stability switching curves and the crossing direction, after which we give the Hopf bifurcation theorem in two-parameter plane for the first time. Under some condition, the intersections of two stability switching curves are double Hopf bifurcation points. To figure out the dynamics near the double Hopf bifurcation point, we calculate the normal form on the center manifold. The derivation process of normal form we use in this paper can be extended to other models with two delays, one delay, or without delay.

\section{INTRODUCTION}

The Leslie-Gower model, one of the most widely used predator-prey models, was proposed by Leslie and Gower [1, 2]

$$
\begin{aligned}
& \dot{u}=r_{1} u\left(1-\frac{u}{K}\right)-a u v, \\
& \dot{v}=r_{2} v\left(1-\frac{v}{\gamma u}\right),
\end{aligned}
$$

where $u(t)$ and $v(t)$ represent the populations of the prey and the predator at time $t$, respectively. $r_{1}$ and $r_{2}$ are the intrinsic growth rates for prey and predator, respectively. $K$ is the environmental carrying capacity for prey population. $a$ is the per capita capturing rate of prey by a predator during unit time. $\frac{v}{\gamma u}$ is Leslie-Gower term with carrying capacity of the predator $\gamma u$, which means that the carrying is proportional to the population size of the prey, and $\gamma$ is referred to as a measure of the quality of the prey as the food for the predator. Since then, various researches on this model and modified models have been carried out. [3 9 ]

Refuges have important effects on the coexistence of predator and prey, and reducing the 
chance of extinction due to predation. Chen et al. [10] incorporated a refuge protecting $m u$ of the prey into Leslie-Gower system, which means that the remaining $(1-m) u$ of the prey is available to the predator. They considered the following Leslie-Gower predator-prey model

$$
\begin{aligned}
& \dot{u}=\left(r_{1}-b_{1} u\right) u-a_{1}(1-m) v u, \\
& \dot{v}=\left[r_{2}-a_{2} \frac{v}{(1-m) u}\right] v,
\end{aligned}
$$

where $m \in[0,1)$ is a refuge protecting rate of the prey.

Time delays are ubiquitous in predator-prey systems. It seems that time delays play an important role in the stability of species densities. Researches are carried out to figure out the effect of delays on predator-prey systems. May [11] considered the feedback time delay in prey growth, and the term $r_{1} u(t)\left(1-\frac{u(t-\tau)}{K}\right)$ is the well-known delayed logistic equation. Another type of time delay was introduced to the negative feedback of the predator's density in Leslie-Gower model in Refs. [12, 13], which denoted the time taken for digestion of the prey. Liu et al. [14] considered both delays mentioned above, and investigated a modified Leslie-Gower predator-prey system with two delays described by the following system:

$$
\left\{\begin{array}{l}
\dot{u}(t)=r_{1} u(t)\left[1-\frac{u\left(t-\tau_{1}\right)}{K}\right]-a(1-m) u(t) v(t), \\
\dot{v}(t)=r_{2} v(t)\left[1-\frac{v\left(t-\tau_{2}\right)}{\gamma(1-m) u\left(t-\tau_{2}\right)}\right]
\end{array}\right.
$$

with the initial conditions

$$
\left(\varphi_{1}, \varphi_{2}\right) \in \mathbf{C}\left([-\tau, 0], \mathbb{R}_{+}^{2}\right), \varphi_{i}(0)>0, i=1,2,
$$

where $\tau_{1}$ is the feedback time delay in prey growth, $\tau_{2}$ is the feedback time delay in predator growth, and we define $\tau=\max \left\{\tau_{1}, \tau_{2}\right\}$.

Related to systems with two delays, general approach is to fix one delay, and vary another, or let $\tau_{1}+\tau_{2}=\tau$. [6, 15 19] However, sometimes, we want to investigate the dynamics of system when two delays vary simultaneously. To discuss systems with two delays, Gu et al. [20] analyzed the characteristic quasipolynomial

$$
p(s)=p_{0}(s)+p_{1}(s) e^{-\tau_{1} s}+p_{2}(s) e^{-\tau_{2} s},
$$

where

$$
p_{l}(s)=\sum_{k=0}^{n} p_{l k} s^{k}
$$


and provided a detailed study on the stability crossing curves such that the characteristic quasipolynomial has at least one imaginary zero and the crossing direction. Lin and Wang [21] considered the following characteristic functions

$$
D\left(\lambda ; \tau_{1}, \tau_{2}\right)=P_{0}(\lambda)+P_{1}(\lambda) e^{-\lambda \tau_{1}}+P_{2}(\lambda) e^{-\lambda \tau_{2}}+P_{3}(\lambda) e^{-\lambda\left(\tau_{1}+\tau_{2}\right)} .
$$

They derived an explicit expression for the stability switching curves in the $\left(\tau_{1}, \tau_{2}\right)$ plane, and gave a criterion to determine switching directions.

Since the preys and predators distribute inhomogenously in different locations, the diffusion should be taken into account in more realistic ecological models. To reveal new phenomena caused by the introduction of inhomogenous spatial environment, Du and Hsu 22] considered a diffusive predator-prey model

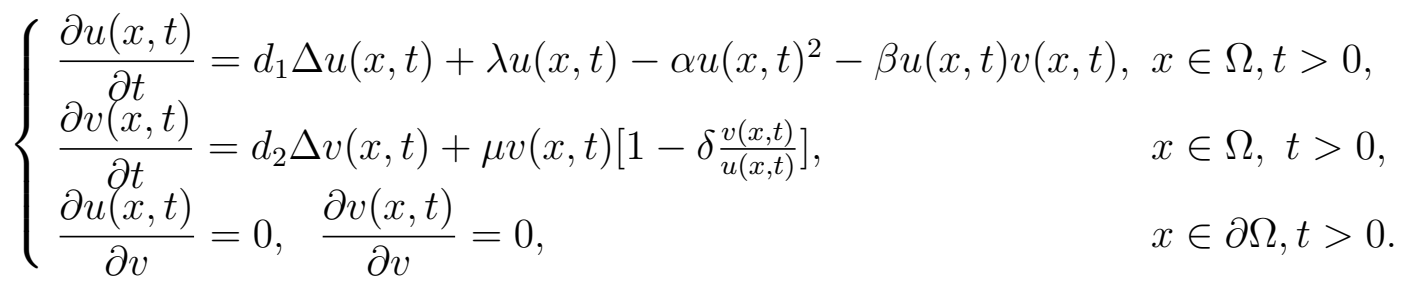

The Neumann boundary condition means that no species can pass across the boundary of $\Omega$. They showed the existence of steady-state solutions with certain prescribed spatial patterns.

Motivated by the previous work, we consider the following modified Leslie-Gower predator-prey model with diffusion and Neumann boundary conditions

$$
\left\{\begin{array}{l}
\frac{\partial u(x, t)}{\partial t}=d_{1} \Delta u(x, t)+r_{1} u(x, t)\left[1-\frac{u\left(x, t-\tau_{1}\right)}{K}\right]-a(1-m) u(x, t) v(x, t), \\
\frac{\partial v(x, t)}{\partial t}=d_{2} \Delta v(x, t)+r_{2} v(x, t)\left[1-\frac{v\left(x, t-\tau_{2}\right)}{\gamma(1-m) u\left(x, t-\tau_{2}\right)}\right], \\
\frac{\partial u(x, t)}{\partial x}=0, \quad \frac{\partial v(x, t)}{\partial x}=0, \text { at } x=0 \text { and } l \pi,
\end{array}\right.
$$

where $d_{1}, d_{2}>0$ are the diffusion coefficients characterizing the rate of the spatial dispersion of the prey and predator population, respectively. $m \in[0,1)$ is a refuge protecting rate of the prey. The spatial interval has been normalized as $[0, l \pi]$.

In fact, there are many literatures on predator-prey model with delays, we refer to Refs. [12, 23 26] and the references therein. Among them, effects of one delay on systems have been discussed widely. In this paper, we focus on the joint effect of two delays on system (6). Adjusting the method given in Ref. [21], which was proposed to solve the analysis of stability switching of delayed differential equations, we can apply the method on reactiondiffusion systems with two delays, and obtain the stability switching results when $\left(\tau_{1}, \tau_{2}\right)$ 
varies simultaneously. To perform bifurcation analysis, we will extend the normal form method given by Faria and Magalhães [27, 28] to the double Hopf bifurcation analysis of the reaction-diffusion system (6), since the normal forms theory, which is an efficient method for bifurcation analysis, can be used to transform the original system to a qualitatively equivalent equation with the simplest form by using near-identity nonlinear transformations.

There are many realistic problems with two delays, such as epidemic model, [29, 30] population interactions, [6, 15, 19, 23, 31] neural networks, [32] coupled oscillators, [33] and so on. As we know, delay usually destabilizes the equilibrium and induces Hopf bifurcation, which gives rise to the periodic activities. For these systems, when we take two delays as parameters, two Hopf bifurcation curves may intersect, and thus double Hopf bifurcation may occur, which is a source of complicated dynamical behaviors. The dynamics near double Hopf bifurcation is much more complicated than those near Hopf bifurcation. We can usually observe rich dynamical behaviors, such as periodic and quasi-periodic oscillations, coexisting of several oscillations, two- or three-dimensional invariant torus, and even chaos. [34, 35] The analysis of double Hopf bifurcation provides a qualitative classification of bifurcating solutions arisen from double Hopf bifurcation, which can help us to figure out the dynamics corresponding to different values of two delays near the double Hopf bifurcation singularity. In fact, the results obtained and the methods used in this paper can be applied to the realistic problems with two delays mentioned above. On one hand, as was pointed out by Lin and Wang, [21] the method of stability switching curves can be used to find the curves where the stability switches, and determine the crossing direction, as long as the characteristic equation of the system we consider has the form of (9). On the other hand, the normal form derivation proposed in this paper can also be used to reaction-diffusion systems with two delays and Neumann boundary conditions, by which the dynamics near the double Hopf singularity can be obtained.

This paper is organized as follows. In section 2, we investigate the stability of the positive equilibrium and the existence of Hopf bifurcation by the method of stability switching curves given in Ref. [21]. In section 3, taking two delays as bifurcation parameters, we derive the normal form on the center manifold near the double Hopf bifurcation point, and give the unfoldings near the critical points. In section 4, we carry out some numerical simulations to support our analytical results. 


\section{STABILITY SWITCHING CURVES AND EXISTENCE OF DOUBLE HOPF BIFURCATION}

In this section, we perform bifurcation analysis near the positive equilibrium $E^{*}$ of system (6). In order to discuss the joint effect of two delays $\tau_{1}$ and $\tau_{2}$ on system (6), we will apply the method of the stability switching curves which is given in Ref. [21].

\subsection{Stability switching curves}

Clearly, system (6) has a unique positive constant equilibrium $E^{*}\left(u^{*}, v^{*}\right)$ with $u^{*}=$ $\frac{K r_{1}}{r_{1}+a K \gamma(1-m)^{2}}$ and $v^{*}=\gamma(1-m) u^{*}$.

The linearization of system (6) at the equilibrium $E^{*}$ is

$$
\frac{\partial}{\partial t}\left(\begin{array}{l}
u(x, t) \\
v(x, t)
\end{array}\right)=(D \Delta+A)\left(\begin{array}{l}
u(x, t) \\
v(x, t)
\end{array}\right)+B\left(\begin{array}{l}
u\left(x, t-\tau_{1}\right) \\
v\left(x, t-\tau_{2}\right)
\end{array}\right)+C\left(\begin{array}{l}
u\left(x, t-\tau_{2}\right) \\
v\left(x, t-\tau_{2}\right)
\end{array}\right)
$$

where

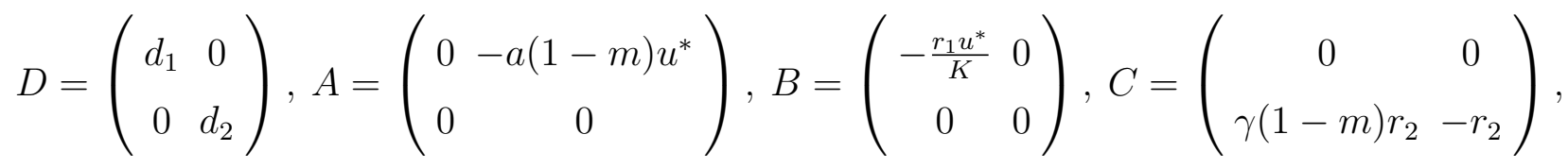

and $u(x, t), v(x, t)$ satisfy the homogeneous Neumann boundary condition.

The characteristic equation of (7) is

$$
\operatorname{det}\left(\lambda I_{2}-M_{n}-A-B e^{-\lambda \tau_{1}}-C e^{-\lambda \tau_{2}}\right)=0,
$$

where $I_{2}$ is the $2 \times 2$ identity matrix and $M_{n}=-\frac{n^{2}}{l^{2}} D, n \in \mathbb{N}_{0}$. The characteristic equation (8) is equivalent to

$$
D_{n}\left(\lambda ; \tau_{1}, \tau_{2}\right)=P_{0, n}(\lambda)+P_{1, n}(\lambda) e^{-\lambda \tau_{1}}+P_{2, n}(\lambda) e^{-\lambda \tau_{2}}+P_{3, n}(\lambda) e^{-\lambda\left(\tau_{1}+\tau_{2}\right)}=0
$$

where

$$
\begin{aligned}
P_{0, n}(\lambda) & =\left(\lambda+d_{1} \frac{n^{2}}{l^{2}}\right)\left(\lambda+d_{2} \frac{n^{2}}{l^{2}}\right), \\
P_{1, n}(\lambda) & =\frac{r_{1}}{K} u^{*}\left(\lambda+d_{2} \frac{n^{2}}{l^{2}}\right), \\
P_{2, n}(\lambda) & =r_{2}\left(\lambda+d_{1} \frac{n^{2}}{l^{2}}\right)+a(1-m)^{2} \gamma r_{2} u^{*}, \\
P_{3, n}(\lambda) & =\frac{r_{1}}{K} u^{*} r_{2} .
\end{aligned}
$$

When $\tau_{1}=\tau_{2}=0$, Eq. (9) becomes

$$
\lambda^{2}+A \lambda+B=0
$$


where

$$
\begin{aligned}
& A=d_{1} \frac{n^{2}}{l^{2}}+d_{2} \frac{n^{2}}{l^{2}}+\frac{r_{1}}{K} u^{*}+r_{2}>0 \\
& B=d_{1} d_{2} \frac{n^{4}}{l^{4}}+\frac{r_{1}}{K} u^{*} d_{2} \frac{n^{2}}{l^{2}}+r_{2} d_{1} \frac{n^{2}}{l^{2}}+a(1-m)^{2} \gamma r_{2} u^{*}+\frac{r_{1}}{K} u^{*} r_{2}>0
\end{aligned}
$$

It is clear that all roots of $(10)$ have negative real parts. Thus, when $\tau_{1}=\tau_{2}=0$, the positive equilibrium $E^{*}\left(u^{*}, v^{*}\right)$ is locally asymptotically stable.

Remark 1. Du and Hsu [22] have proved that the positive equilibrium of the diffusive Leslie-Gower predator-prey system (5) is globally asymptotically stable under certain conditions. We will also mention that Chen et al. [23] have given a global stability result for a diffusive Leslie-Gower predator-prey system with two delays (the delay terms are different from these proposed in this paper). However, delays in this paper will destabilize the equilibrium. Thus, we only give the global stability result for $\tau_{1}=\tau_{2}=0$, which is a direct application of Proposition 2.1 in Ref. [22]. Applying the globally asymptotical stability result in Ref. [22] directly, we have the following result: if $\frac{r_{1}}{K}>a(1-m)$, the positive equilibrium $E^{*}\left(u^{*}, v^{*}\right)$ of (6) is globally asymptotically stable when $\tau_{1}=\tau_{2}=0$.

In order to apply the method of the stability switching curves, [21] we first verify the assumptions (i)-(iv) in Ref. [21] are all true for any fixed $n$.

(i) Finite number of characteristic roots on $\mathbb{C}_{+}=\{\lambda \in \mathbb{C}: \operatorname{Re} \lambda>0\}$ under the condition

$$
\operatorname{deg}\left(P_{0, n}(\lambda)\right) \geq \max \left\{\operatorname{deg}\left(P_{1, n}(\lambda)\right), \operatorname{deg}\left(P_{2, n}(\lambda)\right), \operatorname{deg}\left(P_{3, n}(\lambda)\right)\right\}
$$

(ii) $P_{0, n}(0)+P_{1, n}(0)+P_{2, n}(0)+P_{3, n}(0) \neq 0$.

(iii) $P_{0, n}(\lambda), P_{1, n}(\lambda), P_{2, n}(\lambda), P_{3, n}(\lambda)$ are coprime polynomials.

(iv) $\lim _{\lambda \rightarrow \infty}\left(\left|\frac{P_{1, n}(\lambda)}{P_{0, n}(\lambda)}\right|+\left|\frac{P_{2, n}(\lambda)}{P_{0, n}(\lambda)}\right|+\left|\frac{P_{3, n}(\lambda)}{P_{0, n}(\lambda)}\right|\right)<1$.

In fact, condition (ii)-(iv) are obviously satisfied and (i) follows from Ref. [36]. Thus, similar to Ref. [37], we have the following lemma.

Lemma 1. As the delays $\left(\tau_{1}, \tau_{2}\right)$ vary continuously in $\mathbb{R}_{+}^{2}$, the number of zeros (counting multiplicity) of $D_{n}\left(\lambda ; \tau_{1}, \tau_{2}\right)$ on $\mathbb{C}_{+}$can change only if a zero appears on or cross the imaginary axis. 
To find the stability switching curves, we should seek all the points $\left(\tau_{1}, \tau_{2}\right)$ such that $D_{n}\left(\lambda ; \tau_{1}, \tau_{2}\right)$ has at least one zero on the imaginary axis. Substituting $\lambda=i \omega(\omega>0)$ into (9), we obtain

$$
\left(P_{0, n}(i \omega)+P_{1, n}(i \omega) e^{-i \omega \tau_{1}}\right)+\left(P_{2, n}(i \omega)+P_{3, n}(i \omega) e^{-i \omega \tau_{1}}\right) e^{-i \omega \tau_{2}}=0
$$

From $\left|e^{-i \omega \tau_{2}}\right|=1$, we have

$$
\left|P_{0, n}(i \omega)+P_{1, n}(i \omega) e^{-i \omega \tau_{1}}\right|=\left|P_{2, n}(i \omega)+P_{3, n}(i \omega) e^{-i \omega \tau_{1}}\right|
$$

Thus, we have

$$
\left|P_{0, n}(i \omega)\right|^{2}+\left|P_{1, n}(i \omega)\right|^{2}-\left|P_{2, n}(i \omega)\right|^{2}-\left|P_{3, n}(i \omega)\right|^{2}=2 A_{1, n}(\omega) \cos \left(\omega \tau_{1}\right)-2 B_{1, n}(\omega) \sin \left(\omega \tau_{1}\right),
$$

with

$$
\begin{aligned}
& A_{1, n}(\omega)=\operatorname{Re}\left(P_{2, n}(i \omega) \bar{P}_{3, n}(i \omega)-P_{0, n}(i \omega) \bar{P}_{1, n}(i \omega)\right), \\
& B_{1, n}(\omega)=\operatorname{Im}\left(P_{2, n}(i \omega) \bar{P}_{3, n}(i \omega)-P_{0, n}(i \omega) \bar{P}_{1, n}(i \omega)\right) .
\end{aligned}
$$

If $A_{1, n}(\omega)^{2}+B_{1, n}(\omega)^{2}>0$, there exists a function $\varphi_{1, n}(\omega)$ such that

$$
\begin{aligned}
& A_{1, n}(\omega)=\sqrt{A_{1, n}(\omega)^{2}+B_{1, n}(\omega)^{2}} \cos \left(\varphi_{1, n}(\omega)\right), \\
& B_{1, n}(\omega)=\sqrt{A_{1, n}(\omega)^{2}+B_{1, n}(\omega)^{2}} \sin \left(\varphi_{1, n}(\omega)\right),
\end{aligned}
$$

where, $\varphi_{1, n}(\omega)=\arg \left\{P_{2, n}(i \omega) \bar{P}_{3, n}(i \omega)-P_{0, n}(i \omega) \bar{P}_{1, n}(i \omega)\right\} \in(-\pi, \pi]$. Thus, 11 can be written as

$\left|P_{0, n}(i \omega)\right|^{2}+\left|P_{1, n}(i \omega)\right|^{2}-\left|P_{2, n}(i \omega)\right|^{2}-\left|P_{3, n}(i \omega)\right|^{2}=2 \sqrt{A_{1, n}(\omega)^{2}+B_{1, n}(\omega)^{2}} \cos \left(\varphi_{1, n}(\omega)+\omega \tau_{1}\right)$.

It is obvious that there exists $\tau_{1} \in \mathbb{R}_{+}$satisfying (12) if and only if

$$
\left(\left(\left.P_{0, n}(i \omega)\right|^{2}+\left|P_{1, n}(i \omega)\right|^{2}-\left|P_{2, n}(i \omega)\right|^{2}-\left|P_{3, n}(i \omega)\right|^{2}\right)^{2} \leq 4\left(A_{1, n}(\omega)^{2}+B_{1, n}(\omega)^{2}\right)\right.
$$

Denote the set of $\omega \in \mathbb{R}_{+}$which satisfies (13) as $\Sigma_{n}^{1}$. We notice that (13) also includes the case $A_{1, n}^{2}(\omega)+B_{1, n}^{2}(\omega)=0$.

Denote

$$
\cos \left(\theta_{1, n}(\omega)\right)=\frac{\left|P_{0, n}(i \omega)\right|^{2}+\left|P_{1, n}(i \omega)\right|^{2}-\left|P_{2, n}(i \omega)\right|^{2}-\left|P_{3, n}(i \omega)\right|^{2}}{2 \sqrt{A_{1, n}(\omega)^{2}+B_{1, n}(\omega)^{2}}}, \quad \theta_{1, n} \in[0, \pi]
$$


which leads to

$$
\tau_{1, j_{1}, n}^{ \pm}(\omega)=\frac{ \pm \theta_{1, n}(\omega)-\varphi_{1, n}(\omega)+2 j_{1} \pi}{\omega}, \quad j_{1} \in \mathbb{Z}
$$

Similarly, we have

$$
\tau_{2, j_{2}, n}^{ \pm}(\omega)=\frac{ \pm \theta_{2, n}(\omega)-\varphi_{2, n}(\omega)+2 j_{2} \pi}{\omega}, \quad j_{2} \in \mathbb{Z}
$$

where

$$
\begin{gathered}
\cos \left(\theta_{2, n}(\omega)\right)=\frac{\left|P_{0, n}(i \omega)\right|^{2}-\left|P_{1, n}(i \omega)\right|^{2}+\left|P_{2, n}(i \omega)\right|^{2}-\left|P_{3, n}(i \omega)\right|^{2}}{2 \sqrt{A_{2, n}(\omega)^{2}+B_{2, n}(\omega)^{2}}}, \quad \theta_{2, n} \in[0, \pi], \\
A_{2, n}(\omega)=\operatorname{Re}\left(P_{1, n}(i \omega) \bar{P}_{3, n}(i \omega)-P_{0, n}(i \omega) \bar{P}_{2, n}(i \omega)\right)=2 \sqrt{A_{2, n}(\omega)^{2}+B_{2, n}(\omega)^{2}} \cos \left(\varphi_{2, n}(\omega)\right), \\
\left.B_{2, n}(\omega)=\operatorname{Im}\left(P_{1, n}(i \omega) \bar{P}_{3, n}(i \omega)\right)-P_{0, n}(i \omega) \bar{P}_{2, n}(i \omega)\right)=2 \sqrt{A_{2, n}(\omega)^{2}+B_{2, n}(\omega)^{2}} \sin \left(\varphi_{2, n}(\omega)\right) .
\end{gathered}
$$

Here the condition on $\omega$ is as follows

$$
\left(\left|P_{0, n}(i \omega)\right|^{2}-\left|P_{1, n}(i \omega)\right|^{2}+\left|P_{2, n}(i \omega)\right|^{2}-\left|P_{3, n}(i \omega)\right|^{2}\right)^{2} \leq 4\left(A_{2, n}(\omega)^{2}+B_{2, n}(\omega)^{2}\right)
$$

Denote the set of $\omega \in \mathbb{R}_{+}$which satisfies (16) as $\Sigma_{n}^{2}$. In fact, we can easily show that 13 is equivalent to (16) by squaring both sides of the two conditions (13) and (16). Thus, $\Sigma_{n}^{1}=\Sigma_{n}^{2} \triangleq \Omega_{n}$.

Definition 1. The set

$$
\begin{array}{r}
\Omega_{n}=\left\{\omega \in \mathbb{R}_{+}: F_{n}(\omega) \triangleq\left(\left|P_{0, n}(i \omega)\right|^{2}+\left|P_{1, n}(i \omega)\right|^{2}-\left|P_{2, n}(i \omega)\right|^{2}-\left|P_{3, n}(i \omega)\right|^{2}\right)^{2}\right. \\
\left.-4\left(A_{1, n}(\omega)^{2}+B_{1, n}(\omega)^{2}\right) \leq 0\right\}
\end{array}
$$

is called the crossing set of $D_{n}\left(\lambda ; \tau_{1}, \tau_{2}\right)=0$.

Obviously, when $\omega \in \Omega_{n}$, both $(13)$ and $(16)$ hold. Now we consider the composition of set $\Omega_{n}$.

Lemma 2. The crossing set $\Omega_{n}$ consists of a finite number of intervals of finite length.

Proof. We follow the similar method in Ref. [21] to show this result. Since $F_{n}(\omega)$ is an eighth degree polynomial, and $F_{n}(+\infty)=+\infty, F_{n}(\omega)$ has a finite number of roots on $\mathbb{R}_{+}$.

If $F_{n}(0)>0$, Denote the roots of $F_{n}(\omega)=0$ as $0<a_{1, n}<b_{1, n} \leq a_{2, n}<b_{2, n}<\cdots \leq$ $a_{N, n}<b_{N, n}<+\infty$, and we $\Omega_{n}=\bigcup_{j=1}^{N} \Omega_{j, n}, \quad \Omega_{j, n}=\left[a_{j, n}, b_{j, n}\right]$. 
If $F_{n}(0) \leq 0$, denote the roots of $F_{n}(\omega)$ as $0<b_{1, n} \leq a_{2, n}<b_{2, n}<\cdots \leq a_{N, n}<b_{N, n}<$ $+\infty$, and we have $\Omega_{n}=\bigcup_{j=1}^{N} \Omega_{j, n}, \quad \Omega_{1, n}=\left(0, b_{1, n}\right], \Omega_{j, n}=\left[a_{j, n}, b_{j, n}\right] \quad(j \geq 2)$.

In fact, we can verify that when $\tau_{1}=\tau_{1, j_{1}, n}^{+}(\omega)$, we have $\tau_{2}=\tau_{2, j_{2}, n}^{-}(\omega)$, and when $\tau_{1}=\tau_{1, j_{1}, n}^{-}(\omega)$, we have $\tau_{2}=\tau_{2, j_{2}, n}^{+}(\omega)$. Denote

$$
\begin{aligned}
& \mathcal{T}_{j_{1}, j_{2}, n}^{ \pm j}=\left\{\left(\tau_{1, j_{1}, n}^{ \pm}(\omega), \tau_{2, j_{2}, n}^{\mp}(\omega)\right): \omega \in \Omega_{j, n}\right\} \\
&=\left\{\left(\frac{ \pm \theta_{1, n}(\omega)-\varphi_{1, n}(\omega)+2 j_{1} \pi}{\omega}, \frac{\mp \theta_{2, n}(\omega)-\varphi_{2, n}(\omega)+2 j_{2} \pi}{\omega}\right): \omega \in \Omega_{j, n}\right\} \\
& \mathcal{T}_{n}^{j}=\bigcup_{j_{1}=-\infty}^{\infty} \bigcup_{j_{2}=-\infty}^{\infty}\left(\mathcal{T}_{j_{1}, j_{2}, n}^{+j} \cup \mathcal{T}_{j_{1}, j_{2}, n}^{-j}\right) \cap \mathbb{R}_{+}^{2},
\end{aligned}
$$

and

$$
\mathcal{T}_{n}=\bigcup_{j=1}^{N} \mathcal{T}_{n}^{j}
$$

Definition 2. Any $\left(\tau_{1}, \tau_{2}\right) \in \mathcal{T}_{n}$ is called a crossing point, which makes $D_{n}\left(\lambda ; \tau_{1}, \tau_{2}\right)=0$ have at least one root $i \omega$ with $\omega$ belongs to the crossing set $\Omega_{n}$. The set $\mathcal{T}_{n}$, which is the collection of all the crossing points, is called stability switching curves.

Since $F_{n}\left(a_{j, n}\right)=F_{n}\left(b_{j, n}\right)=0$, we have

$$
\theta_{i, n}\left(a_{j, n}\right)=\delta_{i}^{a} \pi, \quad \theta_{i, n}\left(b_{j, n}\right)=\delta_{i}^{b} \pi
$$

where $\delta_{i}^{a}, \delta_{i}^{b}=0,1, i=1,2$. By (14) and $(15)$, we can easily confirm that

$$
\begin{gathered}
\left(\tau_{1, j_{1}, n}^{+j}\left(a_{j, n}\right), \tau_{2, j_{2}, n}^{-j}\left(a_{j, n}\right)\right)=\left(\tau_{1, j_{1}+\delta_{1}^{a}, n}^{-j}\left(a_{j, n}\right), \tau_{2, j_{2}-\delta_{2}^{a}, n}^{+j}\left(a_{j, n}\right)\right) \\
\left(\tau_{1, j_{1}, n}^{+j}\left(b_{j, n}\right), \tau_{2, j_{2}, n}^{-j}\left(b_{j, n}\right)\right)=\left(\tau_{1, j_{1}+\delta_{1}^{b}, n}^{-j}\left(b_{j, n}\right), \tau_{2, j_{2}-\delta_{2}, n}^{+j}\left(b_{j, n}\right)\right)
\end{gathered}
$$

Thus, for the stability switching curves corresponding to $\Omega_{j, n}, \mathcal{T}_{j_{1}, j_{2}, n}^{+j}$ is connected to $\mathcal{T}_{j_{1}+\delta_{1}^{a}, j_{2}-\delta_{2}^{a}, n}^{-j}$ at one end $a_{j, n}$, and connected to $\mathcal{T}_{j_{1}+\delta_{1}^{b}, j_{2}-\delta_{2}^{b}, n}^{-j}$ at the other end $b_{j, n}$.

\subsection{Crossing directions}

In the following, in order to identify the existence of Hopf bifurcation, we consider the direction in which the root of $(9)$ cross the imaginary axis as $\left(\tau_{1}, \tau_{2}\right)$ deviates from a stability switching curve $\mathcal{T}_{n}^{j}$ by the method given by Lin and Wang. [21] 
Let $\lambda=\sigma+i \omega$. By (9), and the implicit function theorem, $\tau_{1}, \tau_{2}$ can be expressed as function of $\sigma$ and $\omega$. From (9), we have

$$
\begin{aligned}
& \left.\frac{\partial \operatorname{Re} D_{n}\left(\lambda ; \tau_{1}, \tau_{2}\right)}{\partial \sigma}\right|_{\lambda=i \omega}=R_{0},\left.\frac{\partial \operatorname{Im} D_{n}\left(\lambda ; \tau_{1}, \tau_{2}\right)}{\partial \sigma}\right|_{\lambda=i \omega}=I_{0}, \\
& \left.\frac{\partial \operatorname{Re} D_{n}\left(\lambda ; \tau_{1}, \tau_{2}\right)}{\partial \omega}\right|_{\lambda=i \omega}=-I_{0},\left.\frac{\partial \operatorname{Im} D_{n}\left(\lambda ; \tau_{1}, \tau_{2}\right)}{\partial \omega}\right|_{\lambda=i \omega}=R_{0}, \\
& \left.\frac{\partial \operatorname{Re} D_{n}\left(\lambda ; \tau_{1}, \tau_{2}\right)}{\partial \tau_{l}}\right|_{\lambda=i \omega}=R_{l},\left.\frac{\partial \operatorname{Im} D_{n}\left(\lambda ; \tau_{1}, \tau_{2}\right)}{\partial \tau_{l}}\right|_{\lambda=i \omega}=I_{l},
\end{aligned}
$$

where $l=1,2$. By the implicit function theory, if $\operatorname{det}\left(\begin{array}{cc}R_{1} & R_{2} \\ I_{1} & I_{2}\end{array}\right)=R_{1} I_{2}-R_{2} I_{1} \neq 0$. we have

$$
\Delta(\omega):=\left.\left(\begin{array}{cc}
\frac{\partial \tau_{1}}{\partial \sigma} & \frac{\partial \tau_{1}}{\partial \omega} \\
\frac{\partial \tau_{2}}{\partial \sigma} & \frac{\partial \tau_{2}}{\partial \omega}
\end{array}\right)\right|_{\sigma=0, \omega \in \Omega_{n}}=-\left(\begin{array}{cc}
R_{1} & R_{2} \\
I_{1} & I_{2}
\end{array}\right)^{-1}\left(\begin{array}{cc}
R_{0} & -I_{0} \\
I_{0} & R_{0}
\end{array}\right) .
$$

For any stability switching curves $\mathcal{T}_{j_{1}, j_{2}, n}^{ \pm j}$, the direction of the curve corresponding to increasing $\omega \in \Omega_{j, n}$ is called the positive direction, i.e. from $\left(\tau_{1, j_{1}, n}^{ \pm j}\left(a_{j, n}\right), \tau_{2, j_{2}, n}^{\mp j}\left(a_{j, n}\right)\right)$ to $\left(\tau_{1, j_{1}, n}^{ \pm j}\left(b_{j, n}\right), \tau_{2, j_{2}, n}^{\mp j}\left(b_{j, n}\right)\right)$. The region on the left-hand (right-hand) side as we head in the positive directions of the curve is called the region on the left (right). As we have mentioned in the previous section, $\mathcal{T}_{j_{1}, j_{2}, n}^{+j}$ is connected to $\mathcal{T}_{j_{1}+\delta_{1}^{a}, j_{2}-\delta_{2}^{a}, n}^{-j}$ at $a_{j, n}$, then the positive direction of the two curves are opposite. Since the tangent vector of $\mathcal{T}_{j_{1}, j_{2}, n}^{ \pm j}$ at $p^{ \pm}\left(\tau_{1, j_{1}, n}^{ \pm}, \tau_{2, j_{2}, n}^{\mp}\right)$ along the positive direction is $\left.\left(\frac{\partial \tau_{1}}{\partial \omega}, \frac{\partial \tau_{2}}{\partial \omega}\right)\right|_{p^{ \pm}} \triangleq \vec{T}_{p^{ \pm}}$, the normal vector of $\mathcal{T}_{j_{1}, j_{2}, n}^{ \pm j}$ pointing to the right region is $\left.\left(\frac{\partial \tau_{2}}{\partial \omega},-\frac{\partial \tau_{1}}{\partial \omega}\right)\right|_{p^{ \pm}} \triangleq \vec{n}_{p^{ \pm}}$(see Fig. 1). On the other hand, as a pair of complex characteristic roots cross the imaginary axis to the right half plane, $\left(\tau_{1}, \tau_{2}\right)$ moves along the direction $\left.\left(\frac{\partial \tau_{1}}{\partial \sigma}, \frac{\partial \tau_{2}}{\partial \sigma}\right)\right|_{p^{ \pm}}$. We can conclude that if the inner product of these two vectors are positive, i.e.,

$$
\left.\delta(\omega)\right|_{p^{ \pm}}:=\frac{\partial \tau_{1}}{\partial \sigma} \frac{\partial \tau_{2}}{\partial \omega}-\left.\frac{\partial \tau_{2}}{\partial \sigma} \frac{\partial \tau_{1}}{\partial \omega}\right|_{p^{ \pm}}>0
$$

Eq. (9) has two more characteristic roots with positive real parts in the region on the right of $\mathcal{T}_{j_{1}, j_{2}, n}^{ \pm j}$. If the inequality 21 is reversed, then the region on the left 9 has two more characteristic roots with positive real parts.

It is easy to see that $\delta(\omega)=\operatorname{det} \Delta(\omega)$. Since $\operatorname{det}\left(\begin{array}{cc}-R_{0} & I_{0} \\ -I_{0} & -R_{0}\end{array}\right)=R_{0}^{2}+I_{0}^{2} \geq 0,(21)$ can be written as $R_{1} I_{2}-R_{2} I_{1}>0$, if either $R_{0} \neq 0$ or $I_{0} \neq 0$, which is satisfied since we do not consider the case that $i \omega$ is the multiple root of $D_{n}\left(\lambda ; \tau_{1}, \tau_{2}\right)=0$, i.e., $\left.\frac{d D_{n}\left(\lambda ; \tau_{1}, \tau_{2}\right)}{d \lambda}\right|_{\lambda=i \omega}=$ $R_{0}+i I_{0} \neq 0$. 
We can verify that

$$
\begin{aligned}
& R_{1} I_{2}-\left.R_{2} I_{1}\right|_{p^{ \pm}} \\
& =\operatorname{Im}\left\{\overline{-i \omega\left(P_{1, n} e^{-i \omega \tau_{1, j_{1}, n}^{ \pm}}+P_{3, n} e^{-i \omega\left(\tau_{1, j_{1}, n}^{ \pm}+\tau_{2, j_{2}, n}^{\mp}\right)}\right)}(-i \omega)\left(P_{2, n} e^{-i \omega \tau_{2, j_{2}, n}^{\mp}}+P_{3, n} e^{-i \omega\left(\tau_{1, j_{1}, n}^{ \pm}+\tau_{2, j_{2}, n}^{\mp}\right)}\right)\right\} \\
& = \pm \omega^{2}\left|P_{2, n} \bar{P}_{3, n}-P_{0, n} \bar{P}_{1, n}\right| \sin \theta_{1, n} \text {. }
\end{aligned}
$$

Hence,

$$
\left.\delta\left(\omega \in \stackrel{\Omega}{\Omega}_{j, n}\right)\right|_{p^{+}}>0, \quad \forall p^{+} \in \mathcal{T}_{j_{1}, j_{2}, n}^{+j}, \text { and }\left.\delta\left(\omega \in \stackrel{\Omega}{\Omega}_{j, n}\right)\right|_{p^{-}}<0, \quad \forall p^{-} \in \mathcal{T}_{j_{1}, j_{2}, n}^{-j}
$$

since $\theta_{1, n}\left(\AA_{j, n}\right) \subset(0, \pi)$. Here, $\stackrel{\Omega}{\Omega}, n_{j}$ denotes the interior of $\Omega_{j, n}$.

We have the following conclusion.

Lemma 3. For any $j=1,2, \cdots, N$, we have

$$
\delta\left(\omega \in \stackrel{\circ}{\Omega}_{j, n}\right)>0(<0), \quad \forall\left(\tau_{1}(\omega), \tau_{2}(\omega)\right) \in \mathcal{T}_{j_{1}, j_{2}, n}^{+j}\left(\left(\tau_{1}(\omega), \tau_{2}(\omega)\right) \in \mathcal{T}_{j_{1}, j_{2}, n}^{-j}\right) .
$$

Therefore, the region on the right of $\mathcal{T}_{j_{1}, j_{2}, n}^{+j}\left(\mathcal{T}_{j_{1}, j_{2}, n}^{-j}\right)$ has two more (less) characteristic roots with positive real parts.

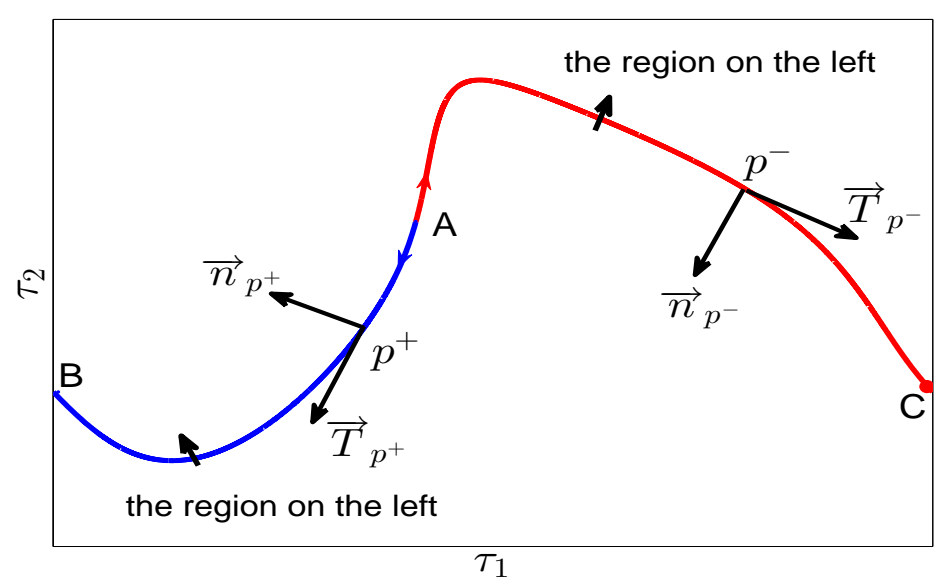

FIG. 1. This is a part of stability switching curves corresponding to $\Omega_{j, n}=\left[a_{j, n}, b_{j, n}\right]$. The blue curve stands for $\mathcal{T}_{j_{1}, j_{2}, n}^{+j}$, with two ends $A\left(\tau_{1, j_{1}, n}^{+j}\left(a_{j, n}\right), \tau_{2, j_{2}, n}^{-j}\left(a_{j, n}\right)\right)$, and $B\left(\tau_{1, j_{1}, n}^{+j}\left(b_{j, n}\right), \tau_{2, j_{2}, n}^{-j}\left(b_{j, n}\right)\right)$. The red curve denotes $\mathcal{T}_{j_{1}+\delta_{1}^{a}, j_{2}-\delta_{2}^{a}, n}^{-j}$, which is connected to $\mathcal{T}_{j_{1}, j_{2}, n}^{+j}$ at $A$ corresponding to $a_{j, n}$, with the positive direction from $A$ to $C$.

We can see from Fig. 1 that the region on the right of $\mathcal{T}_{j_{1}, j_{2}, n}^{+j}$, and the region on the left of $\mathcal{T}_{j_{1}+\delta_{1}^{a}, j_{2}-\delta_{2}^{a}, n}^{-j}$, with the black arrows pointing to, have two more characteristic roots with 
positive real part. Thus, as we move along these curves, stability crossing directions are consistent (see Fig:1, the region with two more characteristic roots with positive real part is on the same side of $\mathcal{T}_{j_{1}, j_{2}, n}^{+j}$ and $\left.\mathcal{T}_{j_{1}+\delta_{1}^{a}, j_{2}-\delta_{2}^{a}, n}^{-j}\right)$.

Any given direction, $\vec{l}=\left(l_{1}, l_{2}\right)$, is pointing to the right region of the curve $\mathcal{T}_{j_{1}, j_{2}, n}^{ \pm j}$, if its inner product with the right-hand side normal $\left(\frac{\partial \tau_{2}}{\partial \omega},-\frac{\partial \tau_{1}}{\partial \omega}\right)$ is positive, i.e.,

$$
l_{1} \frac{\partial \tau_{2}}{\partial \omega}-l_{2} \frac{\partial \tau_{1}}{\partial \omega}>0
$$

And it is pointing to the left region of the curve $\mathcal{T}_{j_{1}, j_{2}, n}^{ \pm j}$ if its inner product with the right-hand side normal is negative.

We have the following result

Corollary 1. As $\left(\tau_{1}, \tau_{2}\right)$ crosses the curve $\mathcal{T}_{j_{1}, j_{2}, n}^{ \pm j}$ along the direction $\vec{l}=\left(l_{1}, l_{2}\right)$, there are two more (less) characteristic roots with positive real parts if

$$
-l_{1}\left(I_{0} I_{1}+R_{0} R_{1}\right)-l_{2}\left(I_{0} I_{2}+R_{0} R_{2}\right)>0(<0) .
$$

Proof. From (20), the left side of (24) becomes

$$
\left[-l_{1}\left(I_{0} I_{1}+R_{0} R_{1}\right)-l_{2}\left(I_{0} I_{2}+R_{0} R_{2}\right)\right] /\left[R_{1} I_{2}-I_{1} R_{2}\right]
$$

If $-l_{1}\left(I_{0} I_{1}+R_{0} R_{1}\right)-l_{2}\left(I_{0} I_{2}+R_{0} R_{2}\right)>0,\left(l_{1}, l_{2}\right)$ is in the same (opposite) side as the righthand side normal of $\mathcal{T}_{j_{1}, j_{2}, n}^{+j}\left(\mathcal{T}_{j_{1}, j_{2}, n}^{-j}\right)$. From Theorem 3 , we can conclude that there are two more characteristic roots with positive real parts of (9) as $\left(\tau_{1}, \tau_{2}\right)$ crosses the curve along the direction $\vec{l}=\left(l_{1}, l_{2}\right)$. we can prove the result similarly when the inequality is reversed.

\subsection{Theorem of Hopf bifurcation}

From the previous discussion, we have the following conclusion about Hopf bifurcation.

Theorem 1. For any $j=1,2, \cdots, N, \mathcal{T}_{n}^{j}$ is a Hopf bifurcation curve in the following sense: for any $p \in \mathcal{T}_{n}^{j}$ and for any smooth curve $\Gamma$ intersecting with $\mathcal{T}_{n}^{j}$ transversely at $p$, we define the tangent of $\Gamma$ at $p$ by $\vec{l}$. If $\left.\frac{\partial \operatorname{Re} \lambda}{\partial \vec{l}}\right|_{p} \neq 0$, and the other eigenvalues of (9) at $p$ have non-zero real parts, then system (6) undergoes a Hopf bifurcation at $p$ when parameters $\left(\tau_{1}, \tau_{2}\right)$ cross $\mathcal{T}_{n}^{j}$ at $p$ along $\Gamma$. 


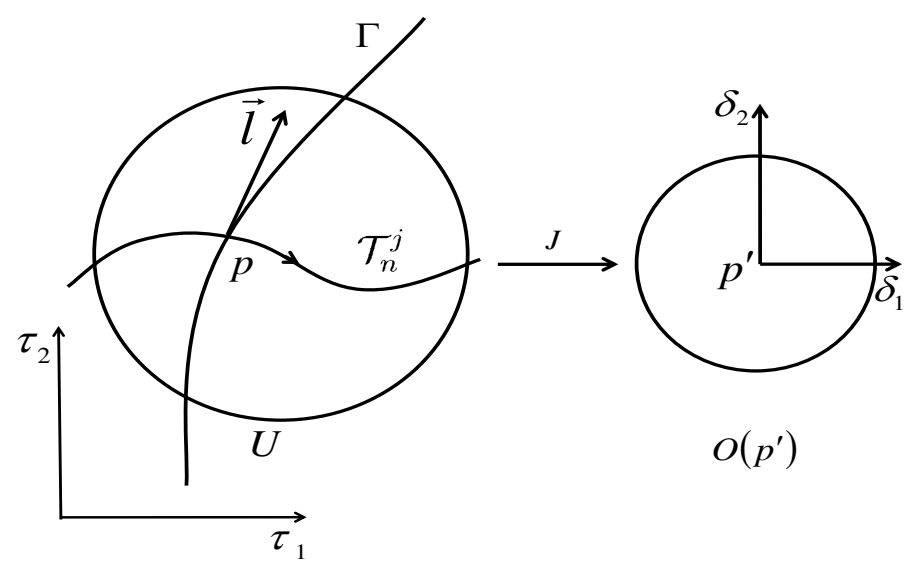

FIG. 2. A sketch of the transformation form $\left(\tau_{1}, \tau_{2}\right)$ plane to $\left(\delta_{1}, \delta_{2}\right)$ plane.

Proof. Denote $p\left(\tau_{1}^{0}, \tau_{2}^{0}\right)$. Let $U$ be a neighbourhood of $p$. Suppose that the equation of curve $\Gamma$ is $\Gamma\left(\tau_{1}, \tau_{2}\right)=0$. Introduce a mapping $J: U \rightarrow \mathbb{R}^{2}$, whose coordinate component function is expressed by $\left\{\begin{array}{l}\delta_{1}=\delta_{1}\left(\tau_{1}, \tau_{2}\right) \\ \delta_{2}=\delta_{2}\left(\tau_{1}, \tau_{2}\right)\end{array}\right.$. Suppose that $J$ locally maps $p\left(\tau_{1}^{0}, \tau_{2}^{0}\right), \mathcal{T}_{n}^{j}$ and $\Gamma$ to $p^{\prime}(0,0), \delta_{1}$ axis and $\delta_{2}$ axis, respectively (shown in Fig. 2), and the Jacobian determinant $\left.\frac{\partial\left(\delta_{1}, \delta_{2}\right)}{\partial\left(\tau_{1}, \tau_{2}\right)}\right|_{p}$ of mapping $J$ is not zero, then by inverse function group theorem, there exists a neighborhood of $p^{\prime}, O\left(p^{\prime}\right)$, such that there is a unique inverse mapping $J^{-1}$ of $J$,

$$
\left\{\begin{array}{l}
\tau_{1}=\tau_{1}\left(\delta_{1}, \delta_{2}\right), \\
\tau_{2}=\tau_{2}\left(\delta_{1}, \delta_{2}\right)
\end{array} \quad\left(\delta_{1}, \delta_{2}\right) \in O\left(p^{\prime}\right)\right.
$$

Now the characteristic equation of system (6) with $\delta_{1}=0$ has purely imaginary root $i \omega$ at $\delta_{2}=0$. We only need to further verify that $\frac{d \operatorname{Re} \lambda}{d \delta_{2}} \neq 0$.

In fact, we have $\delta_{1}=\Gamma\left(\tau_{1}, \tau_{2}\right)$, and the tangent vector of curve $\Gamma$ is $\vec{l}=\left(-\frac{\partial \Gamma}{\partial \tau_{2}}, \frac{\partial \Gamma}{\partial \tau_{1}}\right)^{T}=$ $\left(-\frac{\partial \delta_{1}}{\partial \tau_{2}}, \frac{\partial \delta_{1}}{\partial \tau_{1}}\right)^{T}$. For convenience, denote $J_{1}=\left(\begin{array}{cc}\frac{\partial \delta_{1}}{\partial \tau_{1}} & \frac{\partial \delta_{1}}{\partial \tau_{2}} \\ \frac{\partial \delta_{2}}{\partial \tau_{1}} & \frac{\partial \delta_{2}}{\partial \tau_{2}}\end{array}\right)$. Obviously, $\vec{e}_{\delta_{2}}=(0,1)^{T}=$ $\frac{1}{\operatorname{det} J_{1}} J_{1} \vec{l}$.

Since $\frac{d \operatorname{Re} \lambda}{d \delta_{2}}=\frac{d \sigma}{d \delta_{2}}=\frac{\partial \sigma}{\partial \vec{e}_{\delta_{2}}}=\left(\frac{\partial \sigma}{\partial \delta_{1}}, \frac{\partial \sigma}{\partial \delta_{2}}\right)^{T} \cdot \vec{e}_{\delta_{2}}$, we further treat the inner product as matrix multiplication $\left(\frac{\partial \sigma}{\partial \delta_{1}}, \frac{\partial \sigma}{\partial \delta_{2}}\right) \vec{e}_{\delta_{2}}=\left(\frac{\partial \sigma}{\partial \tau_{1}}, \frac{\partial \sigma}{\partial \tau_{2}}\right) J_{1}^{-1} \frac{1}{\operatorname{det} J_{1}} J_{1} \vec{l}=\frac{1}{\operatorname{det} J_{1}}\left(\frac{\partial \sigma}{\partial \tau_{1}}, \frac{\partial \sigma}{\partial \tau_{2}}\right)^{T} \cdot \vec{l}=\frac{1}{\operatorname{det} J_{1}} \frac{\partial \sigma}{\partial \vec{l}}$. Thus, the transversality condition $\frac{d \operatorname{Re} \lambda}{d \delta_{2}} \neq\left. 0\right|_{\delta_{2}=0}$ holds if $\left.\frac{\partial \operatorname{Re} \lambda}{\partial \vec{l}}\right|_{p} \neq 0$. According to Corollary 2.4 in Ref. [38], the conclusion follows.

Remark 2. Suppose that there exists $\omega_{j_{1}, k_{1}} \in \Omega_{j_{1}, k_{1}}$ and $\omega_{j_{2}, k_{2}} \in \Omega_{j_{2}, k_{2}}$, such that $\mathcal{T}_{k_{1}}^{j_{1}}$ and 
$\mathcal{T}_{k_{2}}^{j_{2}}$ intersect. Then there are two pairs of pure imaginary roots of (9) at the intersection.

Thus, system (6) may undergoes double Hopf bifurcations near the positive equilibrium $E^{*}$ at the intersection of two stability switching curves.

\section{NORMAL FORM ON THE CENTER MANIFOLD FOR DOUBLE HOPF BI- FURCATION}

From the previous section, when two stability switching curves intersect, system (6) may undergoes double Hopf bifurcations near the positive equilibrium $E^{*}$. In order to investigate the dynamical behavior of system (6) near the double Hopf bifurcation point, we will calculate the normal forms of double Hopf bifurcation, by applying the normal form method of partial functional differential equations. [27]

Without loss of generality, we always assume $\tau_{1}>\tau_{2}$ in this section. Otherwise, all the derivations can be proceeded in similar forms. Let $\bar{u}(x, t)=u\left(x, \tau_{1} t\right)-u^{*}, \bar{v}(x, t)=$ $v\left(x, \tau_{1} t\right)-v^{*}$, and drop the bars, system (6) can be written as

$\frac{\partial}{\partial t}\left(\begin{array}{l}u(x, t) \\ v(x, t)\end{array}\right)=\tau_{1}(D \Delta+A)\left(\begin{array}{l}u(x, t) \\ v(x, t)\end{array}\right)+\tau_{1} B\left(\begin{array}{l}u(x, t-1) \\ v(x, t-1)\end{array}\right)+\tau_{1} C\left(\begin{array}{l}u\left(x, t-\tau_{2} / \tau_{1}\right) \\ v\left(x, t-\tau_{2} / \tau_{1}\right)\end{array}\right)+\tau_{1}\left(\begin{array}{c}f_{1} \\ f_{2}\end{array}\right)$,

where

$$
\begin{aligned}
& f_{1}=-\frac{r_{1}}{K} u(x, t) u(x, t-1)-a(1-m) u(x, t) v(x, t), \\
& f_{2}=-\frac{r_{2}}{\gamma(1-m) u^{*}} v(x, t) v\left(x, t-\tau_{2} / \tau_{1}\right)+\frac{r_{2}}{u^{*}} v(x, t) u\left(x, t-\tau_{2} / \tau_{1}\right)+\frac{r_{2}}{u^{*}} v\left(x, t-\tau_{2} / \tau_{1}\right) u\left(x, t-\tau_{2} / \tau_{1}\right) \\
& -\frac{r_{2} \gamma(1-m)}{u^{*}} u^{2}\left(x, t-\tau_{2} / \tau_{1}\right)+\frac{r_{2}}{\gamma(1-m) u^{* 2}} v(x, t) u\left(x, t-\tau_{2} / \tau_{1}\right) v\left(x, t-\tau_{2} / \tau_{1}\right) \\
& -\frac{r_{2}}{u^{* 2}} v(x, t) u^{2}\left(x, t-\tau_{2} / \tau_{1}\right)-\frac{r_{2}}{u^{* 2}} v\left(x, t-\tau_{2} / \tau_{1}\right) u^{2}\left(x, t-\tau_{2} / \tau_{1}\right)+\frac{r_{2} \gamma(1-m)}{u^{* 2}} u^{3}\left(x, t-\tau_{2} / \tau_{1}\right) .
\end{aligned}
$$

For the Neumann boundary condition, we define the real-valued Hilbert space

$$
X=\left\{(u, v)^{T} \in H^{2}(0, l \pi) \times H^{2}(0, l \pi): \frac{\partial u}{\partial x}=\frac{\partial v}{\partial x}=0 \text { at } x=0, l \pi\right\}
$$

and the corresponding complexification space of $X$ by $X_{\mathbb{C}}:=X \oplus i X=\left\{U_{1}+i U_{2}: U_{1}, U_{2} \in\right.$ $X\}$, with the general complex-value $L^{2}$ inner product $\langle U, V\rangle=\int_{0}^{l \pi}\left(\bar{u}_{1} v_{1}+\bar{u}_{2} v_{2}\right) d x$, for $U=\left(u_{1}, u_{2}\right)^{T}, V=\left(v_{1}, v_{2}\right)^{T} \in X_{\mathbb{C}}$. Let $\mathscr{C}:=C\left([-1,0], X_{\mathbb{C}}\right)$ denote the phase space with the sup norm. We write $u^{t} \in \mathscr{C}$ for $u^{t}(\theta)=u(t+\theta),-1 \leq \theta \leq 0$. 
Denote the double Hopf bifurcation point by $\left(\tau_{1}^{*}, \tau_{2}^{*}\right)$. Introduce two bifurcation parameters $\sigma=\left(\sigma_{1}, \sigma_{2}\right)$ by setting $\sigma_{1}=\tau_{1}-\tau_{1}^{*}, \sigma_{2}=\tau_{2}-\tau_{2}^{*}$, and denote $U(t)=(u(t), v(t))^{T}$, then (27) can be written as

$$
\frac{d U(t)}{d t}=D\left(\tau_{1}^{*}+\sigma_{1}, \tau_{2}^{*}+\sigma_{2}\right) \Delta U(t)+L\left(\tau_{1}^{*}+\sigma_{1}, \tau_{2}^{*}+\sigma_{2}\right)\left(U^{t}\right)+F\left(\tau_{1}^{*}+\sigma_{1}, \tau_{2}^{*}+\sigma_{2}, U^{t}\right),
$$

where

$$
\begin{aligned}
& D\left(\tau_{1}^{*}+\sigma_{1}, \tau_{2}^{*}+\sigma_{2}\right)=\left(\tau_{1}^{*}+\sigma_{1}\right) D=\tau_{1}^{*} D+\sigma_{1} D, \\
& L\left(\tau_{1}^{*}+\sigma_{1}, \tau_{2}^{*}+\sigma_{2}\right) U^{t} \\
& =\left(\tau_{1}^{*}+\sigma_{1}\right) A U^{t}(0)+\left(\tau_{1}^{*}+\sigma_{1}\right) B U^{t}(-1)+\left(\tau_{1}^{*}+\sigma_{1}\right) C U^{t}\left(-\left(\tau_{2}^{*}+\sigma_{2}\right) /\left(\tau_{1}^{*}+\sigma_{1}\right)\right) \\
& =\tau_{1}^{*}\left(A U^{t}(0)+B U^{t}(-1)+C U^{t}\left(-\tau_{2}^{*} / \tau_{1}^{*}\right)\right)+\sigma_{1}\left(A U^{t}(0)+B U^{t}(-1)+C U^{t}\left(-\tau_{2}^{*} / \tau_{1}^{*}\right)\right) \\
& +\tau_{1}^{*} C\left(U^{t}\left(-\left(\tau_{2}^{*}+\sigma_{2}\right) /\left(\tau_{1}^{*}+\sigma_{1}\right)\right)-U^{t}\left(-\tau_{2}^{*} / \tau_{1}^{*}\right)\right)+\sigma_{1} C\left(U^{t}\left(-\left(\tau_{2}^{*}+\sigma_{2}\right) /\left(\tau_{1}^{*}+\sigma_{1}\right)\right)-U^{t}\left(-\tau_{2}^{*} / \tau_{1}^{*}\right)\right), \\
& F\left(\tau_{1}^{*}+\sigma_{1}, \tau_{2}^{*}+\sigma_{2}, U^{t}\right)=\left(\tau_{1}^{*}+\sigma_{1}\right)\left(f_{1}, f_{2}\right)^{T} .
\end{aligned}
$$

Consider the linearized system of (28)

$$
\frac{d U(t)}{d t}=\tau_{1}^{*} D \Delta U(t)+\tau_{1}^{*}\left(A U^{t}(0)+B U^{t}(-1)+C U^{t}\left(-\tau_{2}^{*} / \tau_{1}^{*}\right)\right) \triangleq D_{0} \Delta U(t)+L_{0}\left(U^{t}\right) .
$$

It is well known that the eigenvalues of $D \Delta$ on $X$ are $-d_{1} \frac{n^{2}}{l^{2}}$ and $-d_{2} \frac{n^{2}}{l^{2}}, n \in \mathbb{N}_{0}=$ $\{0,1,2, \cdots\}$, with corresponding normalized eigenfunctions $\beta_{n}^{1}(x)=\gamma_{n}(x)(1,0)^{T}$ and $\beta_{n}^{2}(x)=\gamma_{n}(x)(0,1)^{T}$, where $\gamma_{n}(x)=\frac{\cos \frac{n}{l} x}{\left\|\cos \frac{n}{l} x\right\|_{L^{2}}}$. Define $\mathscr{B}_{n}$ the subspace of $\mathscr{C}$, by $\mathscr{B}_{n}:=\operatorname{span}\left\{\left\langle v(\cdot), \beta_{n}^{j}\right\rangle \beta_{n}^{j} \mid v \in \mathscr{C}, j=1,2\right\}$, satisfying $L\left(\mathscr{B}_{n}\right)\left(\tau_{1}, \tau_{2}\right) \subset \operatorname{span}\left\{\beta_{n}^{1}, \beta_{n}^{2}\right\}$. For simplification of notations, we write $\left\langle v(\cdot), \beta_{n}\right\rangle=\left(\left\langle v(\cdot), \beta_{n}^{1}\right\rangle,\left\langle v(\cdot), \beta_{n}^{2}\right\rangle\right)^{T}$.

System 28 can be written as

$$
\frac{d U(t)}{d t}=D_{0} \Delta U(t)+L_{0}\left(U^{t}\right)+G\left(\sigma, U^{t}\right)
$$

where

$$
\begin{aligned}
G\left(\sigma, U^{t}\right) & =\sigma_{1}\left(D \Delta U^{t}(0)+A U^{t}(0)+B U^{t}(-1)+C U^{t}\left(-\tau_{2}^{*} / \tau_{1}^{*}\right)\right) \\
& +\left(\tau_{1}^{*}+\sigma_{1}\right) C\left(U^{t}\left(-\left(\tau_{2}^{*}+\sigma_{2}\right) /\left(\tau_{1}^{*}+\sigma_{1}\right)\right)-U^{t}\left(-\tau_{2}^{*} / \tau_{1}^{*}\right)\right) \\
& +F\left(\tau_{1}^{*}+\sigma_{1}, \tau_{2}^{*}+\sigma_{2}, U^{t}\right) .
\end{aligned}
$$

Rewrite (30) as an abstract ordinary differential equation on $\mathscr{C}$ [27]

$$
\frac{d}{d t} U^{t}=A U^{t}+X_{0} G\left(\sigma, U^{t}\right)
$$

where $A$ is the infinitesimal generator of the $C_{0}$-semigroup of solution maps of the linear equation (28), defined by $A: \mathscr{C}_{0}^{1} \cap \mathscr{C} \rightarrow \mathscr{C}, A \varphi=\dot{\varphi}+X_{0}\left[D_{0} \Delta \varphi(0)+L_{0}(\varphi)-\dot{\varphi}(0)\right]$, with 
$\operatorname{dom}(A)=\{\varphi \in \mathscr{C}: \dot{\varphi} \in \mathscr{C}, \varphi(0) \in \operatorname{dom}(\Delta)\}$, and $X_{0}$ is given by $X_{0}(\theta)=0$ for $\theta \in[-1,0)$ and $X_{0}(0)=I$.

Then on $\mathscr{B}_{n}$, the linear equation $\frac{d}{d t} U(t)=D_{0} \Delta U(t)+L_{0}\left(U^{t}\right)$ is equivalent to the retarded functional differential equation on $\mathbb{C}^{2}: \dot{z}(t)=-\frac{n^{2}}{l^{2}} D_{0} z(t)+L_{0} z^{t}$. Define functions of bounded variation $\eta_{m} \in B V([-1,0], \mathbb{R})$ such that

$$
-\frac{k_{m}^{2}}{l^{2}} D_{0} \varphi(0)+L_{0}(\varphi)=\int_{-1}^{0} d \eta_{m}(\theta) \varphi(\theta), \varphi \in \mathscr{C} .
$$

Let $A_{m}(m=1,2)$ denote the infinitesimal generator of the semigroup generated by (3), and $A_{m}^{*}$ denote the formal adjoint of $A_{m}$ under the bilinear form

$$
(\alpha, \beta)_{m}=\alpha(0) \beta(0)-\int_{-1}^{0} \int_{0}^{\theta} \alpha(\xi-\theta) d \eta_{m}(\theta) \beta(\xi) d \xi .
$$

From the previous section, we know that system (29) has two pairs of pure imaginary eigenvalues $\pm i \omega_{j_{1}, k_{1}} \tau_{1}^{*}, \pm i \omega_{j_{2}, k_{2}} \tau_{1}^{*}$ at the double Hopf bifurcation point and the other eigenvalues with nonzero real parts. For simplicity of notation, we denote them by $\left\{ \pm i \omega_{1} \tau_{1}^{*}, \pm i \omega_{2} \tau_{1}^{*}\right\}$. Suppose that $\omega_{1}: \omega_{2} \neq m: n$ for $m, n \in \mathbb{N}$ and $1 \leq m, n \leq 3$, i.e., we do not consider the strongly resonant cases. Using the formal adjiont theory, we decompose $\mathscr{C}$ by $\left\{ \pm i \omega_{1} \tau_{1}^{*}, \pm i \omega_{2} \tau_{1}^{*}\right\}$ as $\mathscr{C}=P_{m} \oplus Q_{m}$, where $Q_{m}=\left\{\phi \in \mathscr{C}:(\psi, \phi)_{m}=0\right.$, for $\left.\psi \in P_{m}^{*}\right\}$, $m=1,2$. We choose the basis $\Phi_{1}(\theta)=\left(\phi_{1}(\theta), \bar{\phi}_{1}(\theta)\right), \Phi_{2}(\theta)=\left(\phi_{3}(\theta), \bar{\phi}_{3}(\theta), \Psi_{1}(s)=\right.$ $\left(\psi_{1}(s), \bar{\psi}_{1}(s)\right)^{T}, \Psi_{2}(s)=\left(\psi_{3}(s), \bar{\psi}_{3}(s)\right)^{T}$, in $P_{1}, P_{1}^{*}, P_{2}, P_{2}^{*}$, satisfying $\left(\Psi_{m}, \Phi_{m}\right)_{m}=I$, and

$$
A_{m} \Phi_{m}=\Phi_{m} B_{m}, A_{m}^{*} \Psi_{m}=B_{m} \Psi_{m}, m=1,2
$$

with $B_{1}=\operatorname{diag}\left(i \omega_{1} \tau_{1}^{*},-i \omega_{1} \tau_{1}^{*}\right), B_{2}=\operatorname{diag}\left(i \omega_{2} \tau_{1}^{*},-i \omega_{2} \tau_{1}^{*}\right)$. Denote $\Phi(\theta)=\left(\Phi_{1}(\theta), \Phi_{2}(\theta)\right)$, and $\Psi(s)=\left(\Psi_{1}(s), \Psi_{2}(s)\right)^{T}$. By a few calculations, we have

$$
\begin{array}{r}
\phi_{1}(\theta)=\left(1, r_{12}\right)^{T} e^{i \omega_{1} \tau_{1}^{*} \theta}, \phi_{3}(\theta)=\left(1, r_{32}\right)^{T} e^{i \omega_{2} \tau_{1}^{*} \theta} \\
\psi_{1}(s)=D_{1}\left(1, r_{12}^{*}\right) e^{-i \omega_{1} \tau_{1}^{*} s}, \psi_{3}(s)=D_{2}\left(1, r_{32}^{*}\right) e^{-i \omega_{2} \tau_{1}^{*} s},
\end{array}
$$

where

$$
\begin{aligned}
& r_{12}=\frac{\gamma(1-m) r_{2} e^{-i \omega_{1} \tau_{2}^{*}}}{r_{2} e^{-i \omega_{1} \tau_{2}^{*}}+d_{2} \frac{n^{2}}{l 2}+i \omega_{1}}, \quad r_{32}=\frac{\gamma(1-m) r_{2} e^{-i \omega_{2} \tau_{2}^{*}}}{r_{2} e^{-i \omega_{2} \tau_{2}^{*}+d_{2} \frac{n^{2}}{l^{2}}+i \omega_{2}}}, \\
& r_{12}^{*}=\frac{-a(1-m) u^{*}}{r_{2} e^{-i \omega_{1} \tau_{2}^{*}}+d_{2} \frac{n^{2}}{l^{2}}+i \omega_{1}}, \quad r_{32}^{*}=\frac{-a(1-m) u^{*}}{r_{2} e^{-i \omega_{2} \tau_{2}^{*}+d_{2} \frac{n^{2}}{l^{2}}+i \omega_{2}}}, \\
& D_{1}=\frac{1}{1+r_{12}^{*} r_{12}-\tau_{1}^{*} \frac{r_{1}}{K} u^{*} e^{-i \omega_{1} \tau_{1}^{*}}+r_{12}^{*} \gamma(1-m) r_{2} \tau_{2}^{*} e^{-i \omega_{1} \tau_{2}^{*}-r_{2} \tau_{2}^{*} e^{-i \omega_{1} \tau_{2}^{*}} r_{12}^{*} r_{12}}}, \\
& D_{2}=\frac{1}{1+r_{32}^{*} r_{32}-\tau_{1}^{*} \frac{r_{1}}{K} u^{*} e^{-i \omega_{2} \tau_{1}^{*}}+r_{32}^{*} \gamma(1-m) r_{2} \tau_{2}^{*} e^{-i \omega_{2} \tau_{2}^{*}-r_{2} \tau_{2}^{*} e^{-i \omega_{2} \tau_{2}^{*}} r_{32}^{*} r_{32}}} .
\end{aligned}
$$


Now, we can decompose $\mathscr{C}$ into a center subspace and its orthocomplement, i.e.,

$$
\mathscr{C}=\mathcal{P} \oplus \operatorname{Ker} \pi
$$

where $\pi: \mathscr{C} \rightarrow \mathcal{P}$ is the projection defined by $\pi(\varphi)=\sum_{m=1}^{2} \Phi_{m}\left(\Psi_{m},\left\langle\varphi(\cdot), \beta_{k_{m}}\right\rangle\right)_{m} \cdot \beta_{k_{m}}$, with $\beta_{k_{m}}=\left(\beta_{k_{m}}^{1}, \beta_{k_{m}}^{2}\right), m=1,2$.

Define the enlarged phase space [27] $\mathscr{B} \mathscr{C}:=\left\{\psi:[-1,0] \rightarrow X_{\mathbb{C}}: \psi\right.$ is continuous on $[-1,0)$, $\left.\exists \lim _{\theta \rightarrow 0^{-}} \psi(\theta) \in X_{\mathbb{C}}\right\}$.

According to (33), $U^{t} \in X$ can be composed as

$$
U^{t}(\theta)=\phi_{1}(\theta) z_{1} \gamma_{k_{1}}+\bar{\phi}_{1}(\theta) z_{2} \gamma_{k_{1}}+\phi_{3}(\theta) z_{3} \gamma_{k_{2}}+\bar{\phi}_{3}(\theta) z_{4} \gamma_{k_{2}}+w(\theta) \triangleq \Phi(\theta) z_{x}+w(\theta),
$$

where $w \in \mathscr{C}^{1} \bigcap \operatorname{Ker} \pi:=\mathcal{Q}^{1}$ for any $t$. Then in $\mathscr{B} \mathscr{C}$ the system (31) is equivalent to the system

$$
\begin{aligned}
& \dot{z_{1}}=i \omega_{1} \tau_{1}^{*} z_{1}+\psi_{1}(0)\left\langle G\left(\sigma, \Phi(\theta) z_{x}+w(\theta)\right), \beta_{k_{1}}\right\rangle, \\
& \dot{z_{2}}=-i \omega_{1} \tau_{1}^{*} z_{2}+\bar{\psi}_{1}(0)\left\langle G\left(\sigma, \Phi(\theta) z_{x}+w(\theta)\right), \beta_{k_{1}}\right\rangle, \\
& \dot{z_{3}}=i \omega_{2} \tau_{1}^{*} z_{3}+\psi_{3}(0)\left\langle G\left(\sigma, \Phi(\theta) z_{x}+w(\theta)\right), \beta_{k_{2}}\right\rangle, \\
& \dot{z_{4}}=-i \omega_{2} \tau_{1}^{*} z_{1}+\bar{\psi}_{3}(0)\left\langle G\left(\sigma, \Phi(\theta) z_{x}+w(\theta)\right), \beta_{k_{2}}\right\rangle, \\
& \frac{d w}{d t}=A_{1} w+(I-\pi) X_{0} G\left(\sigma, \Phi(\theta) z_{x}+w(\theta)\right),
\end{aligned}
$$

where $A_{1}$ is the restriction of $A$ on $\mathcal{Q}^{1} \subset \operatorname{Ker} \pi \rightarrow \operatorname{Ker} \pi, A_{1} \varphi=A \varphi$ for $\varphi \in \mathcal{Q}^{1}$.

Consider the formal Taylor expansion

$$
G(\sigma, \varphi)=\frac{1}{2 !} G_{2}(\sigma, \varphi)+\frac{1}{3 !} G_{3}(\sigma, \varphi)
$$

where $G_{j}$ is the $j$ th Fréchet derivation of $G$, which we calculate in section 1 of supplement material. Then 35 can be written as

$$
\begin{aligned}
& \dot{z}=B z+\sum_{j \geq 2} \frac{1}{j !} f_{j}^{1}(z, w, \sigma), \\
& \frac{d}{d t} w=A_{1} w+\sum_{j \geq 2} \frac{1}{j !} f_{j}^{2}(z, w, \sigma),
\end{aligned}
$$

where $z=\left(z_{1}, z_{2}, z_{3}, z_{4}\right) \in \mathbb{C}^{4}, w \in \mathcal{Q}^{1}, B=\operatorname{diag},\left(B_{1}, B_{2}\right)=\operatorname{diag}\left(\mathrm{i} \omega_{1} \tau_{1}^{*},-\mathrm{i} \omega_{1} \tau_{1}^{*}, \mathrm{i} \omega_{2} \tau_{1}^{*},-\mathrm{i} \omega_{2} \tau_{1}^{*}\right)$, and $f_{j}=\left(f_{j}^{1}, f_{j}^{2}\right), j \geq 2$, are defined by

$$
\begin{aligned}
& f_{j}^{1}(z, w, \sigma)=\left(\begin{array}{l}
\psi_{1}(0)\left\langle G_{j}\left(\sigma, \Phi(\theta) z_{x}+w(\theta)\right), \beta_{k_{1}}\right\rangle \\
\bar{\psi}_{1}(0)\left\langle G_{j}\left(\sigma, \Phi(\theta) z_{x}+w(\theta)\right), \beta_{k_{1}}\right\rangle \\
\psi_{3}(0)\left\langle G_{j}\left(\sigma, \Phi(\theta) z_{x}+w(\theta)\right), \beta_{k_{2}}\right\rangle \\
\bar{\psi}_{3}(0)\left\langle G_{j}\left(\sigma, \Phi(\theta) z_{x}+w(\theta)\right), \beta_{k_{2}}\right\rangle
\end{array}\right), \\
& f_{j}^{2}=(I-\pi) X_{0} G_{j}\left(\sigma, \Phi(\theta) z_{x}+w(\theta)\right),
\end{aligned}
$$


Similar as in Ref. [27], and using the notations in it, define the operator $M_{j}=\left(M_{j}^{1}, M_{j}^{2}\right)$, $j \geq 2$ by

$$
\begin{array}{ll}
M_{j}^{1}: V_{j}^{4+2}\left(\mathbb{C}^{4}\right) \rightarrow V_{j}^{4+2}\left(\mathbb{C}^{4}\right), & \left(M_{j}^{1} p\right)(z, \sigma)=D_{z} p(z, \sigma) B z-B p(z, \sigma), \\
M_{j}^{2}: V_{j}^{4+2}\left(\mathcal{Q}_{1}\right) \subset V_{j}^{4+2}(\operatorname{Ker} \pi) \rightarrow V_{j}^{4+2}(\operatorname{Ker} \pi), & \left(M_{j}^{2} h\right)(z, \sigma)=D_{z} h(z, \sigma) B z-A_{1} h(z, \sigma) .
\end{array}
$$

When $\omega_{1}: \omega_{2} \neq m: n$ for $m, n \in \mathbb{N}$ and $1 \leq m, n \leq 3$ (i.e., the case of nonresonant double Hopf bifurcation) it is easy to verify that

$$
\begin{aligned}
& \operatorname{Im}\left(M_{2}^{1}\right)^{c}=\operatorname{span}\left\{\sigma_{1} z_{1} e_{1}, \sigma_{2} z_{1} e_{1}, \sigma_{1} z_{2} e_{2}, \sigma_{2} z_{2} e_{2}, \sigma_{1} z_{3} e_{3}, \sigma_{2} z_{3} e_{3}, \sigma_{1} z_{4} e_{4}, \sigma_{2} z_{4} e_{4}\right\} . \\
& \operatorname{Im}\left(M_{3}^{1}\right)^{c}=\operatorname{span}\left\{z_{1}^{2} z_{2} e_{1}, z_{1} z_{3} z_{4} e_{1}, z_{1} z_{2}^{2} e_{2}, z_{2} z_{3} z_{4} e_{2}, z_{3}^{2} z_{4} e_{3} z_{1} z_{2} z_{3} e_{3}, z_{3} z_{4}^{2} e_{4}, z_{1} z_{2} z_{4} e_{4}\right\} .
\end{aligned}
$$

where $e_{1}=(1,0,0,0)^{T}, e_{2}=(0,1,0,0)^{T}, e_{3}=(0,0,1,0)^{T}, e_{4}=(0,0,0,1)^{T}$. According to Ref. [27], by a recursive transformations of variables of the following form

$$
(z, w, \sigma)=(\widehat{z}, \widehat{w}, \sigma)+\frac{1}{j !}\left(U_{j}^{1}(\widehat{z}, \sigma), U_{j}^{2}(\widehat{z}, \sigma), 0\right)
$$

with $U_{j}=\left(U_{j}^{1}, U_{j}^{2}\right) \in V_{j}^{4+2}\left(\mathbb{C}^{4}\right) \times V_{j}^{4+2}\left(\mathcal{Q}_{1}\right)$, [27] which transforms 36 into the normal form

$$
\begin{aligned}
& \dot{z}=B z+\sum_{j \geq 2} \frac{1}{j !} g_{j}^{1}(z, w, \sigma), \\
& \frac{d w}{d t}=A_{1} w+\sum_{j \geq 2} \frac{1}{j !} g_{j}^{2}(z, w, \sigma),
\end{aligned}
$$

where $g_{j}=\left(g_{j}^{1}, g_{j}^{2}\right), j \geq 2$, are given by $g_{j}(z, w, \sigma)=\bar{f}_{j}(z, w, \sigma)-M_{j} U_{j}(z, \sigma)$, and $U_{j} \in$ $V_{j}^{4+2}\left(\mathbb{C}^{4}\right) \times V_{j}^{4+2}\left(\mathcal{Q}_{1}\right)$ are expressed as

$$
U_{j}(z, \sigma)=\left(M_{j}\right)^{-1} \operatorname{Proj}_{\operatorname{Im}\left(M_{j}^{1}\right) \times \operatorname{Im}\left(M_{j}^{2}\right)} \circ \bar{f}_{j}(z, 0, \sigma)
$$

where $\bar{f}_{j}=\left(\bar{f}_{j}^{1}, \bar{f}_{j}^{2}\right)$ stand for the terms of order $j$ in $(z, w)$, which are obtained after the computation of normal forms up to order $j-1$. From Ref. [27], the normal form truncated to the third order has the following form

$$
\dot{z}=B z+\frac{1}{2 !} g_{2}^{1}(z, 0, \sigma)+\frac{1}{3 !} g_{3}^{1}(z, 0,0)+\text { h.o.t. }
$$

Here $g_{3}^{1}(z, 0,0)=\operatorname{Proj}_{\operatorname{Ker}\left(M_{3}^{1}\right)} \bar{f}_{3}^{1}(z, 0,0)$, where

$$
\begin{gathered}
\bar{f}_{3}^{1}(z, 0,0)=f_{3}^{1}(z, 0,0)+\frac{3}{2}\left[D_{z} f_{2}^{1}(z, 0,0) U_{2}^{1}(z, 0)\right. \\
\left.+D_{w} f_{2}^{1}(z, 0,0) U_{2}^{2}(z, 0)-D_{z} U_{2}^{1}(z, 0) g_{2}^{1}(z, 0,0)\right]
\end{gathered}
$$


and $\left(U_{2}^{1}(z, \sigma), U_{2}^{2}(z, \sigma)\right) \in V_{j}^{4+2}\left(\mathbb{C}^{4}\right) \times V_{j}^{4+2}\left(\mathcal{Q}_{1}\right)$ given by 41 .

The calculations of $g_{2}^{1}(z, 0, \sigma)$ and $g_{3}^{1}(z, 0,0)$ heavily depends on tedious mathematical derivations such as solve the center manifold function and some projections, thus we leave the details in section 2 and 3 of the supplement materials.

Finally, the normal form truncated to the third order on the center manifold for double Hopf bifurcation is obtained as follows

$$
\begin{aligned}
& \dot{z}_{1}=i \omega_{1} \tau_{1}^{*} z_{1}+K_{11} \sigma_{1} z_{1}+K_{21} \sigma_{2} z_{1}+K_{2100} z_{1}^{2} z_{2}+K_{1011} z_{1} z_{3} z_{4}, \\
& \dot{z}_{2}=-i \omega_{1} \tau_{1}^{*} z_{2}+\overline{K_{11}} \sigma_{1} z_{2}+\overline{K_{21}} \sigma_{2} z_{2}+\overline{K_{2100}} z_{1} z_{2}^{2}+\overline{K_{1011}} z_{2} z_{3} z_{4}, \\
& \dot{z}_{3}=i \omega_{2} \tau_{1}^{*} z_{3}+K_{13} \sigma_{1} z_{3}+K_{23} \sigma_{2} z_{3}+K_{0021} z_{3}^{2} z_{4}+K_{1110} z_{1} z_{2} z_{3}, \\
& \dot{z}_{4}=-i \omega_{2} \tau_{1}^{*} z_{4}+\overline{K_{13}} \sigma_{1} z_{4}+\overline{K_{23}} \sigma_{2} z_{4}+\overline{K_{0021}} z_{3} z_{4}^{2}+\overline{K_{1110}} z_{1} z_{2} z_{4}
\end{aligned}
$$

Make the polar coordinate transformation

$$
\begin{aligned}
& z_{1}=\rho_{1} \cos \theta_{1}+i \rho_{1} \sin \theta_{1}, \quad z_{2}=\rho_{1} \cos \theta_{1}-i \rho_{1} \sin \theta_{1}, \\
& z_{3}=\rho_{2} \cos \theta_{2}+i \rho_{2} \sin \theta_{2}, \quad z_{4}=\rho_{2} \cos \theta_{2}-i \rho_{2} \sin \theta_{2},
\end{aligned}
$$

where $\rho_{1}, \rho_{2}>0$. Denote $\epsilon_{1}=\operatorname{Sign}\left(\operatorname{Re} K_{2100}\right), \epsilon_{2}=\operatorname{Sign}\left(\operatorname{Re} K_{0021}\right)$, rescale as $\widehat{\rho}_{1}=$

$\rho_{1} \sqrt{\left|K_{2100}\right|}, \widehat{\rho}_{2}=\rho_{2} \sqrt{\left|K_{0021}\right|}, \widehat{t}=t \epsilon_{1}$, and drop the hats, then we obtain the equivalent system of (44)

$$
\begin{aligned}
& \dot{\rho}_{1}=\rho_{1}\left(\nu_{1}+\rho_{1}^{2}+b \rho_{2}^{2}\right), \\
& \dot{\rho}_{2}=\rho_{2}\left(\nu_{2}+c \rho_{1}^{2}+d \rho_{2}^{2}\right) .
\end{aligned}
$$

Here

$$
\begin{aligned}
& \nu_{1}=\epsilon_{1}\left(\operatorname{Re} K_{11} \sigma_{1}+\operatorname{Re} K_{21} \sigma_{2}\right), \nu_{2}=\epsilon_{1}\left(\operatorname{Re} K_{13} \sigma_{1}+\operatorname{Re} K_{23} \sigma_{2}\right), \\
& b=\frac{\epsilon_{1} \epsilon_{2} \operatorname{Re} K_{1011}}{\operatorname{Re} K_{0021}}, c=\frac{\operatorname{Re} K_{1110}}{\operatorname{Re} K_{2100}}, d=\epsilon_{1} \epsilon_{2} .
\end{aligned}
$$

As was discussed in chapter 7.5 in Ref. [35], there are twelve distinct kinds of unfoldings for Eq. 45) (see Table 1). In section 4, case VIa arises, thus we draw bifurcation set and phase portraits for the unfolding of case VIa in Fig. 3.

\section{NUMERICAL SIMULATIONS}

In this section, we carry out some simulations. We choose

$$
r_{1}=0.8, r_{2}=1, a=1.3, K=0.7, \gamma=1, m=0.27, l=2, d_{1}=0.3, d_{2}=0.4 \text {, }
$$


TABLE I. The twelve unfoldings of system (45).

\begin{tabular}{lllllllllllll}
\hline Case & Ia & Ib & II & III & IVa & IVb & V & VIa & VIb & VIIa & VIIb & VIII \\
\hline$d$ & +1 & +1 & +1 & +1 & +1 & +1 & -1 & -1 & -1 & -1 & -1 & -1 \\
$b$ & + & + & + & - & - & - & + & + & + & - & - & - \\
$c$ & + & + & - & + & - & - & + & - & - & + & + & - \\
$d-b c$ & + & - & + & + & + & - & - & + & - & + & - & - \\
\hline
\end{tabular}

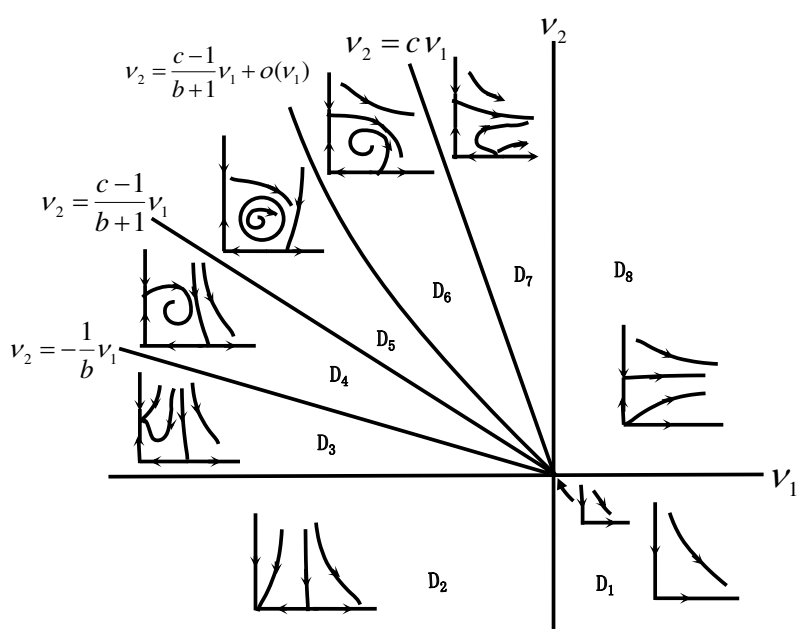

FIG. 3. Phase portraits for the unfoldings of case VIa with $\epsilon_{1}=1$.

then one can get the unique positive constant equilibrium $E^{*}(0.4358,0.3181)$, which is globally asymptotically stable when $\tau_{1}=\tau_{2}=0$ according to Remark 1 .

To illustrate the dynamics in the presence of delays, we follow the process given in section 2.1. As shown in Fig. 4 a), $F_{0}(0)>0$, and $F_{0}(\omega)=0$ has four roots $a_{1,0}=$ $0.2587, b_{1,0}=0.6682, a_{2,0}=0.7697$, and $b_{2,0}=1.1791$. The crossing set is $\Omega_{1,0} \bigcup \Omega_{2,0}=$ $\left[a_{1,0}, b_{1,0}\right] \cup\left[a_{2,0}, b_{2,0}\right]$. For the two ends of the $\Omega_{1,0}$, we have $\theta_{1,0}\left(a_{1,0}\right)=\pi, \theta_{2,0}\left(a_{1,0}\right)=\pi$, $\theta_{1,0}\left(b_{1,0}\right)=\pi$, and $\theta_{2,0}\left(b_{1,0}\right)=0$, and $\delta_{1}^{a}=1, \delta_{2}^{a}=1, \delta_{1}^{b}=1, \delta_{2}^{b}=0$. From (17) and (18), we can get the stability switching curves $\mathcal{T}_{0}^{1}$ corresponding to $\Omega_{1,0}$ which is shown in Fig. 4 b). From the previous discussion in $\left(19,, \mathcal{T}_{j_{1}, j_{2}, 0}^{+1}\right.$ is connected to $\mathcal{T}_{j_{1}+1, j_{2}-1,0}^{-1}$ at the left point $a_{1,0}$ and $\mathcal{T}_{j_{1}+1, j_{2}, 0}^{-1}$ at the right point $b_{1,0}$ for any $j_{1}, j_{2}$. To show the structure of stability switching curves and the crossing direction clearly, we take the left-most curve of $\mathcal{T}_{0}^{1}$ (i.e. $\tau_{0}^{1(1)}$ in Fig. 4 b)) as an example, and draw the figure in Fig. 5 a). From bottom to top, 
a)

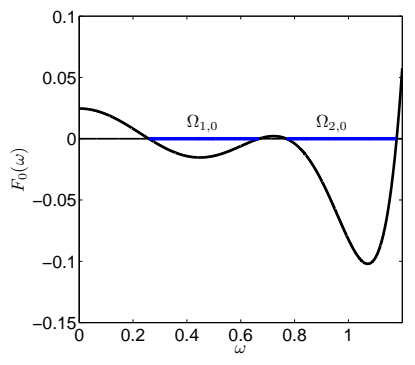

b)

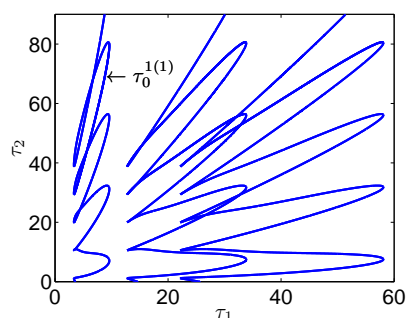

c)

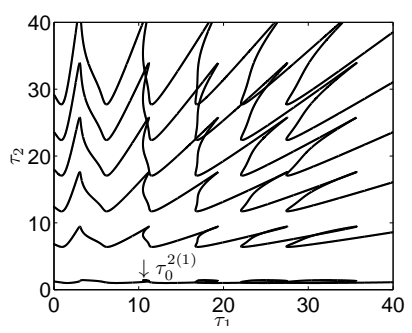

FIG. 4. a) Graph of $F_{0}(\omega)$. b) Stability switching curves $\mathcal{T}_{0}^{1}$. c) Stability switching curves $\mathcal{T}_{0}^{2}$.

a)

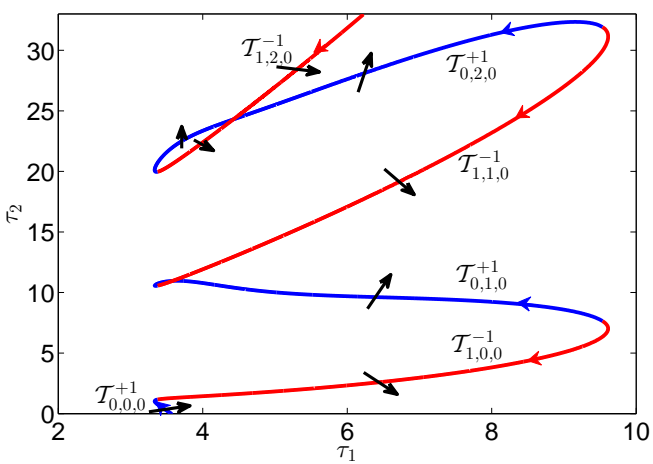

b)

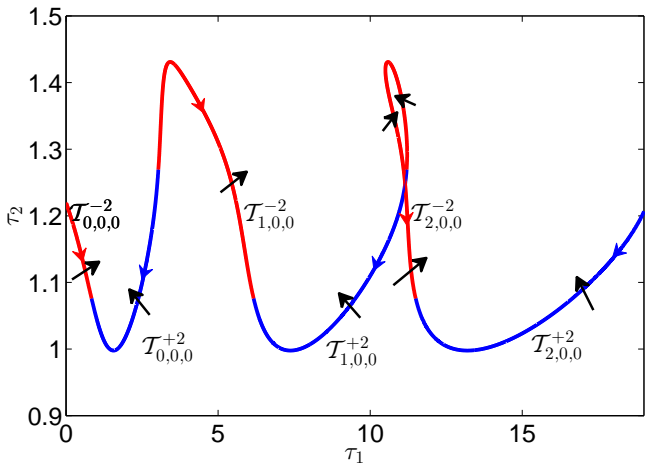

FIG. 5. a) The detailed structure of the left-most curve of $\mathcal{T}_{0}^{1}$ (marked $\tau_{0}^{1(1)}$ in Fig. 4 b)). The blue (red) arrow represents the positive direction of $\mathcal{T}_{j_{1}, j_{2}, 0}^{+1}\left(\mathcal{T}_{j_{1}, j_{2}, 0}^{-1}\right)$. b) The detailed structure of the lowest curve of $\mathcal{T}_{0}^{2}$ (marked $\tau_{0}^{2(1)}$ in Fig. 4 c)). The blue (red) arrow represents the positive direction of $\mathcal{T}_{j_{1}, j_{2}, 0}^{+2}\left(\mathcal{T}_{j_{1}, j_{2}, 0}^{-2}\right)$. From Lemma 3, we know that the regions on the right (left) of the blue (red) curves, which the black arrows point to, have two more characteristic roots with positive real parts.

it starts with a part of $\mathcal{T}_{0,0,0}^{+1}$, which is connected to $\mathcal{T}_{1,0,0}^{-1}$ at $b_{1,0} . \mathcal{T}_{1,0,0}^{-1}$ is linked to $\mathcal{T}_{0,1,0}^{+1}$ at $a_{1,0}$, which is again connected to $\mathcal{T}_{1,1,0}^{-1}$ at $b_{1,0} \cdots \cdots$. The numerical results coincide with the analysis result in (19). In fact, the rest curves of $\mathcal{T}_{0}^{1}$ in Fig. 4 b)) have similar structure as $\tau_{0}^{1(1)}$. Similarly, the stability switching curves $\mathcal{T}_{0}^{2}$ corresponding to $\Omega_{2,0}$ are shown in Fig. 4 c), and the lowest curve of $\mathcal{T}_{0}^{2}\left(\operatorname{marked} \tau_{0}^{2(1)}\right.$ in Fig. 4 c) ) is drawn in Fig. 5 b). All the stability switching curves for $n=0$ are given by $\mathcal{T}_{0}=\mathcal{T}_{0}^{1} \cup \mathcal{T}_{0}^{2}$.

When $n=1, F_{1}(0)>0$, and $F_{1}(\omega)=0$ has four roots $a_{1,1}=0.184, b_{1,1}=0.5264, a_{2,1}=$ $0.8607, b_{2,1}=1.189$, which is shown in Fig. 6 a). Thus, the crossing set is $\Omega_{1,1} \bigcup \Omega_{2,1}=$ $\left[a_{1,1}, b_{1,1}\right] \cup\left[a_{2,1}, b_{2,1}\right]$, and we can get the stability switching curves $\mathcal{T}_{1}^{1}$ and $\mathcal{T}_{1}^{2}$, which is shown in Fig. 6 b) and c). Thus all the stability switching curves for $n=1$ are given by 
a)

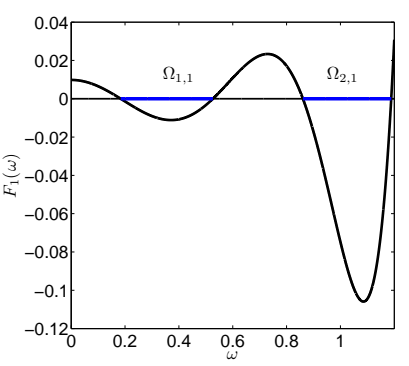

b)

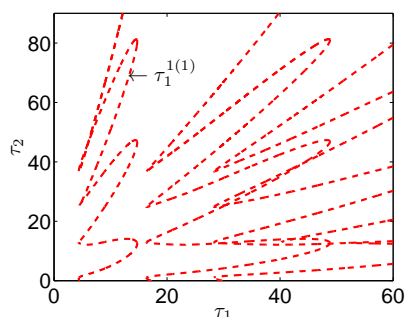

c)

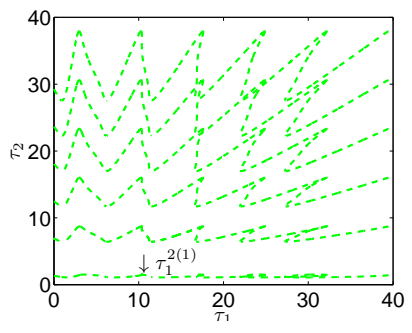

FIG. 6. a) Graph of $F_{1}(\omega)$. b) Stability switching curves $\mathcal{T}_{1}^{1}$. c) Stability switching curves $\mathcal{T}_{1}^{2}$.

a)

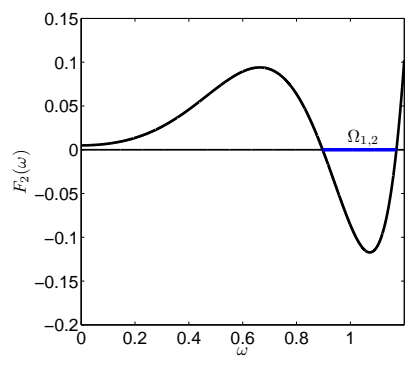

b)

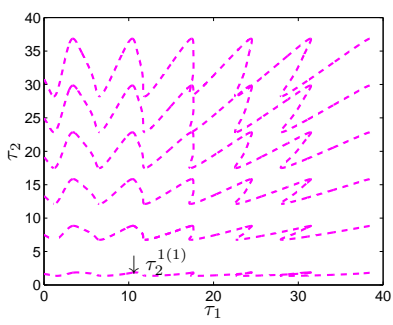

FIG. 7. a) Graph of $F_{2}(\omega)$. b) Stability switching curves $\mathcal{T}_{2}^{1}$.

$\mathcal{T}_{1}=\mathcal{T}_{1}^{1} \cup \mathcal{T}_{1}^{2}$

When $n=2, F_{2}(0)>0$, and $F_{2}(\omega)=0$ has two roots $a_{1,2}=0.8968, b_{1,2}=1.171$, which is shown in Fig. 7 a). The crossing set is $\Omega_{1,2}=\left[a_{1,2}, b_{1,2}\right]$. The stability switching curves $\mathcal{T}_{2}^{1}$ corresponding to $\Omega_{1,2}$ is shown in Fig. $7 \mathrm{~b}$ ). All the stability switching curves for $n=2$ are given by $\mathcal{T}_{2}=\mathcal{T}_{2}^{1}$.

When $n=3, F_{3}(0)>0$, and $F_{3}(\omega)=0$ has two roots $a_{1,3}=0.6638, b_{1,3}=0.9798$, which is shown in Fig. 8 a). The crossing set is $\Omega_{1,3}=\left[a_{1,3}, b_{1,3}\right]$. And the stability switching curves $\mathcal{T}_{3}^{1}$ corresponding to $\Omega_{1,3}$ is shown in Fig. $8 \mathrm{~b}$ ). Thus all the stability switching curves for $n=3$ are given by $\mathcal{T}_{3}=\mathcal{T}_{3}^{1}$.

When $n \geq 4$, numerical calculation indicates $F_{n}(\omega)>0$ for any $\omega$, thus there are no stability switching curves on $\left(\tau_{1}, \tau_{2}\right)$ plane for $n \geq 4$.

Combining the stability switching curves shown in Fig. 4, together, and zooming in the part when $\left(\tau_{1}, \tau_{2}\right) \in[0,5] \times[0,3]$, we have the Hopf bifurcation curves shown in Fig. 9 a). We focus on the bottom left region bounded by left-most curve of $\mathcal{T}_{0}^{1}$ and the lowest curve of $\mathcal{T}_{0}^{2}$, which is shown in Fig. 9 a). Notice that the left-most and the lowest curve among all the stability switching curves are both part of $\mathcal{T}_{0}$. By Theorem 3 , we 
a)

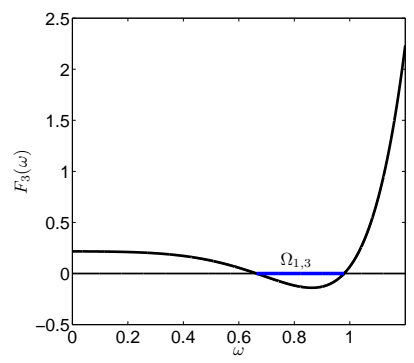

b)

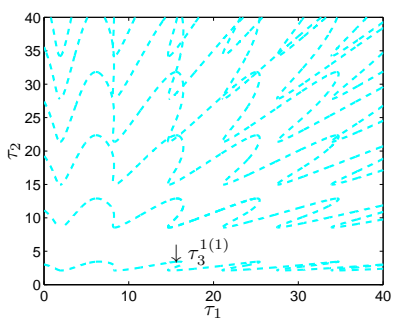

FIG. 8. a) Graph of $F_{3}(\omega)$. b) Stability switching curves $\mathcal{T}_{3}^{1}$.

a)

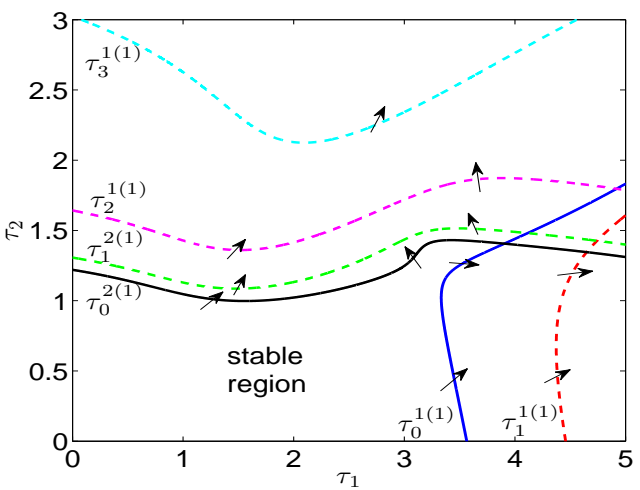

b)

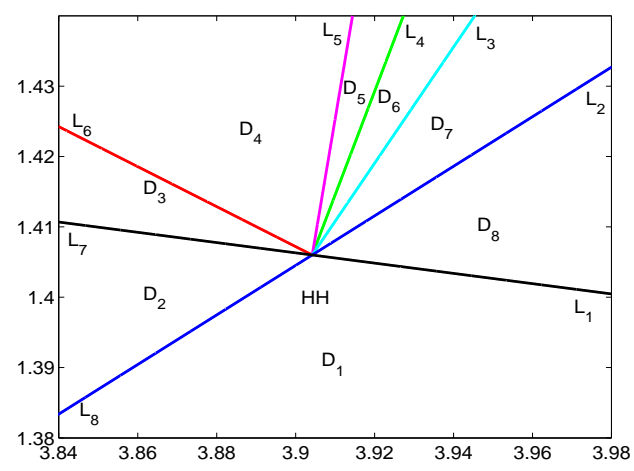

FIG. 9. a) The left-most curve and the lowest curve of $\mathcal{T}_{0}$ intersect at $\left(\tau_{1}, \tau_{2}\right)=(3.9042,1.406)$, which is a double Hopf bifurcation point on the $\tau_{1}-\tau_{2}$ plane. Crossing directions are marked by arrows. b) The complete bifurcation sets near HH.

can verify that the positive equilibrium $E^{*}$ is stable in the bottom left region, since the crossing directions of the two switching curves (the black line and blue line) are all pointing outside of the region. Moreover, we can see that the two stability switching curves intersect at the point $(3.9042,1.406)$, and we denote the double Hopf bifurcation point by $\mathrm{HH}$. For HH, using the normal form derivation process given in section 3, we have $\omega_{1}=0.61081, \omega_{2}=0.94964, K_{11}=0.0947-0.0071 i, K_{21}=-0.2689+0.4408 i, K_{13}=$ $0.1196+1.2137 i, K_{23}=1.6381-2.5531 i, K_{2100}=0.0154-0.0146 i, K_{1011}=0.4878+$ $0.2082 i, K_{0021}=-0.9861-0.9526 i, K_{1110}=-0.1778-0.1523 i$. Furthermore, we have the normal form (45) with $\epsilon_{1}=1, \epsilon_{2}=-1, b=0.4946, c=-11.5623, d=-1$, and $d-b c=4.7192$.

This means that the unfolding system near the double Hopf bifurcation point $\mathrm{HH}$ is of type VIa. According to Guckenheimer and Holmes, [35] near the double Hopf bifurcation 


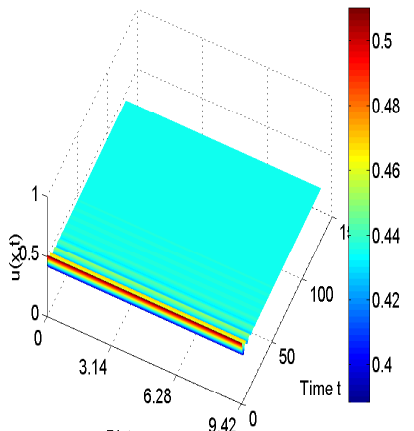

a) Distance $x \quad 9.42$ b)

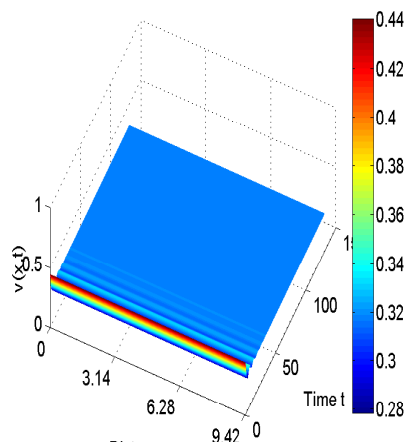

Distance $x \quad 9.42$

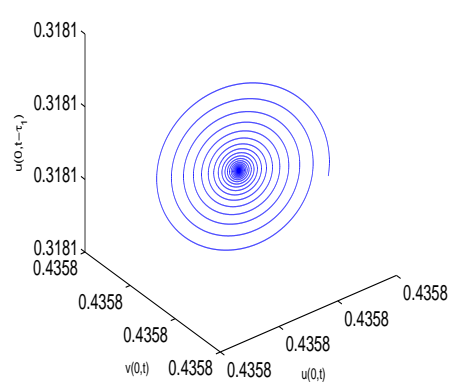

c)

FIG. 10. When $\tau_{1}=1.74, \tau_{2}=0.67$ in $D_{2}$, the positive equilibrium is asymptotically stable.

point there are eight different kinds of phase diagrams in eight different regions which are divided by semi-lines L1-L8 with

$$
\begin{aligned}
& L_{1}: \tau_{2}=\left(\tau_{1}-3.9042\right) /(-13.6972)+1.406 \quad\left(\tau_{1}>3.9042\right) \\
& L_{2}: \tau_{2}=\left(\tau_{1}-3.9042\right) /(2.8383)+1.406 \quad\left(\tau_{1}>3.9042\right) \\
& L_{3}: \tau_{2}=\left(\tau_{1}-3.9042\right) /(1.2106)+1.406 \quad\left(\tau_{2}>1.406\right) \\
& L_{4}: \tau_{2}=\left(\tau_{1}-3.9042\right) /(0.6790)+1.406+o\left(\tau_{1}-3.9042\right) \quad\left(\tau_{2}>1.406\right) ; \\
& L_{5}: \tau_{2}=\left(\tau_{1}-3.9042\right) /(0.6790)+1.406 \quad\left(\tau_{2}>1.406\right) ; \\
& L_{6}: \tau_{2}=\left(\tau_{1}-3.9042\right) /(-3.5180)+1.406 \quad\left(\tau_{2}>1.406\right) ; \\
& L_{7}: \tau_{2}=\left(\tau_{1}-3.9042\right) /(-13.6972)+1.406 \quad\left(\tau_{1}<3.9042\right) \\
& L_{8}: \tau_{2}=\left(\tau_{1}-3.9042\right) /(2.8381)+1.406 \quad\left(\tau_{1}<3.9042\right) .
\end{aligned}
$$

According to Fig. 3, we have the bifurcation set near HH showing in Fig. 9 b). It is found that $\mathrm{HH}$ is the intersection of a supercritical Hopf bifurcation curve and a subcritical Hopf bifurcation curve.

When $\tau_{1}=1.74, \tau_{2}=0.67$ in $D_{2}$, the positive equilibrium is a sink, which is shown in Fig. 10. When $\tau_{1}=3.62, \tau_{2}=1.435$ in region $D_{3}$, there is a stable periodic solutions originating from a supercritical Hopf bifurcation, which is shown in Fig. 11.

Finally, we show the existence of quasi-periodic solutions and the results of the Poincaré map on a Poincaré section. Due to the fact that the double Hopf bifurcation point is the intersection of two curves from $\mathcal{T}_{0}$, all periodic or quasi-periodic solutions nearby are spatially homogeneous. Thus, we choose the solution curve of $(u(0, t), v(0, t))$ at $x=0$ to show the rich dynamics. Since the periodic solutions oscillate in an infinite dimensional 


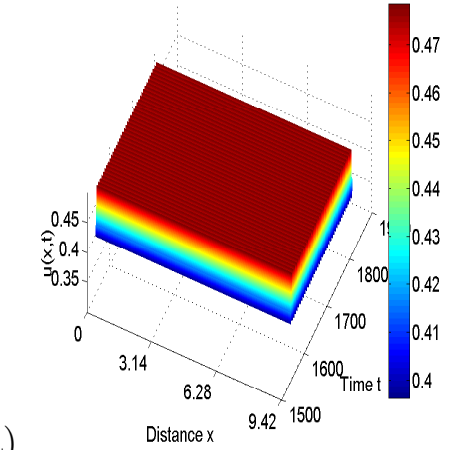

a) Distancex $\quad 9.421500 \quad$ b)

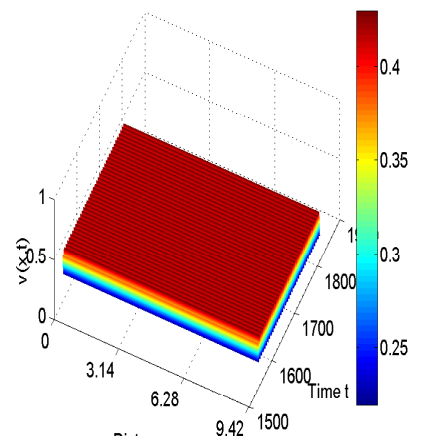

Distance $x \quad 9.421500$

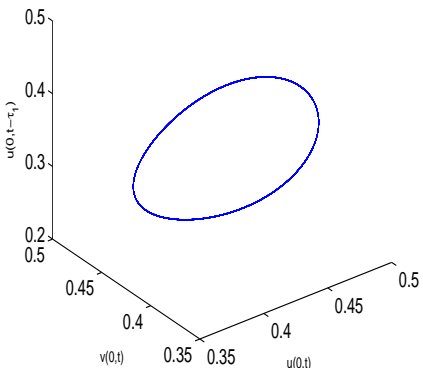

c)

FIG. 11. When $\tau_{1}=3.62, \tau_{2}=1.435$ in $D_{3}$, there is a stable periodic solution.

phase space, [38] we give simulations near the double Hopf bifurcation point in the space $u(0, t)-v(0, t)-u\left(0, t-\tau_{1}\right)$ and choose the Poincaré sections $v(0, t)=v^{*}, \dot{u}(0, t)=0$, respectively, in Fig. 12. The system exhibits rich dynamical behavior near the bifurcation point. When $\left(\tau_{1}, \tau_{2}\right)=(3.82,1.4345)$ in $D_{4}$, system (6) has a quasi-periodic solution on a 2-torus (Fig. 12 a)). It becomes a quasi-periodic solution on a 3-torus, which breaks down through an orbit-connection bifurcation at $\left(\tau_{1}, \tau_{2}\right)=(3.9043,1.418)$ ( Fig. $\left.12 \mathrm{~b}\right)$ ). When the parameters vary and enter $D_{6}$, three-dimensional torus vanish. Due to the fact that a vanishing 3-torus might accompany the phenomenon of chaos, [39] 41] near the double Hopf bifurcation point, we find strange attractors exist. We can see that when $\tau_{1}=3.905, \tau_{2}=$ 1.4136 in region $D_{6}$, system (6) has a strange attractor, which is shown on the Poincaré section in Fig. 12 c).

\section{CONCLUDING REMARKS}

This paper deals with a modified Leslie-Gower predator-prey system with two delays and diffusion. We focus on the joint effect of two delays on the dynamical behavior of the system. Applying the method of stability switching curves, we find the stable region of the positive equilibrium and obtain Hopf bifurcation results. By searching the intersection of stability switching curves near the stable region, we get the double Hopf bifurcation point. Through the calculation of normal form of the system, we get the corresponding unfolding system and the bifurcation set. We theoretically prove and illustrate the existence of quasi-periodic solution on two-torus, quasi-periodic solution on three-torus, and even strange attractor.

The theorem of Hopf bifurcation corresponding to systems with single parameter has 
a)
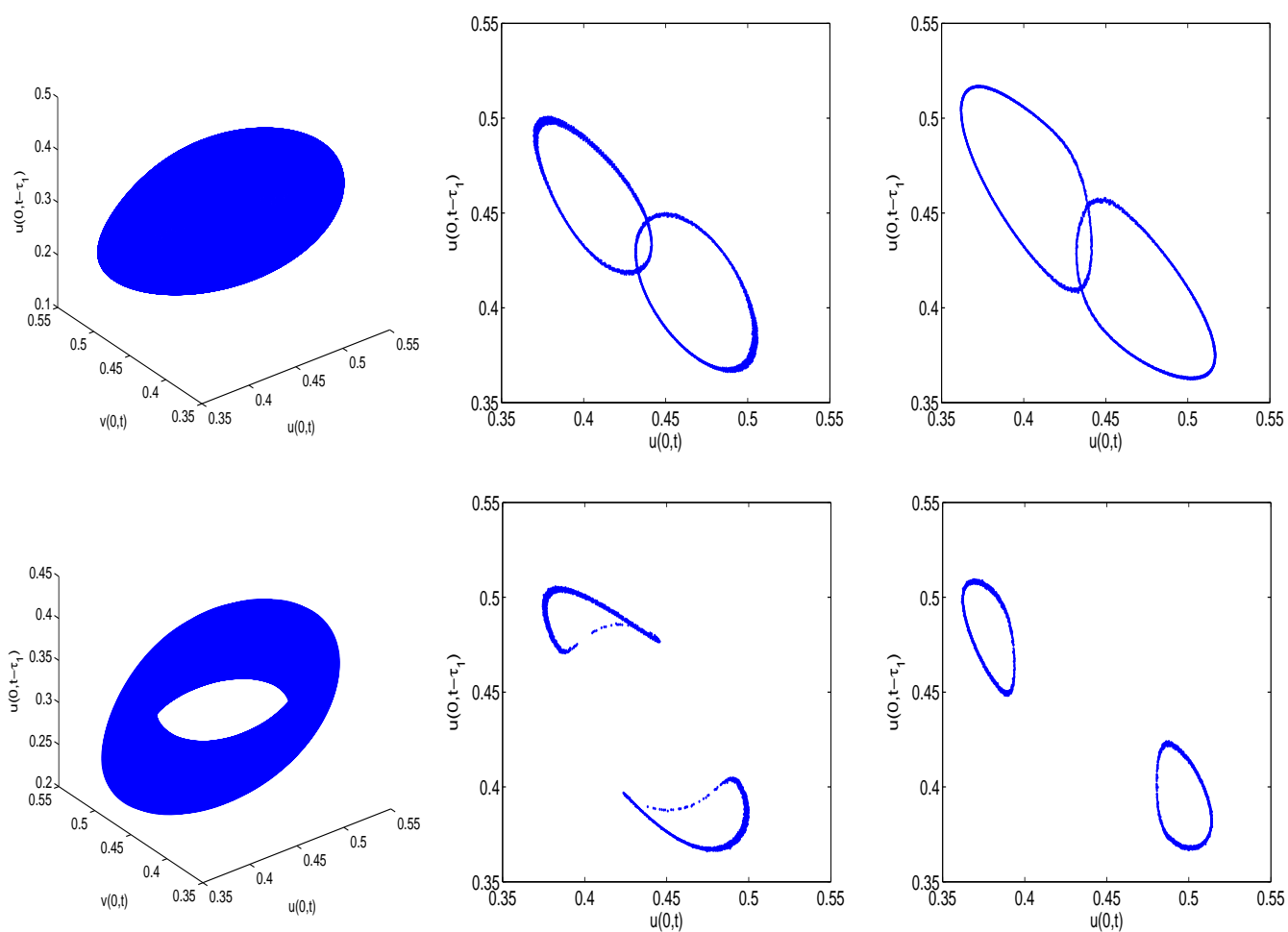

b)
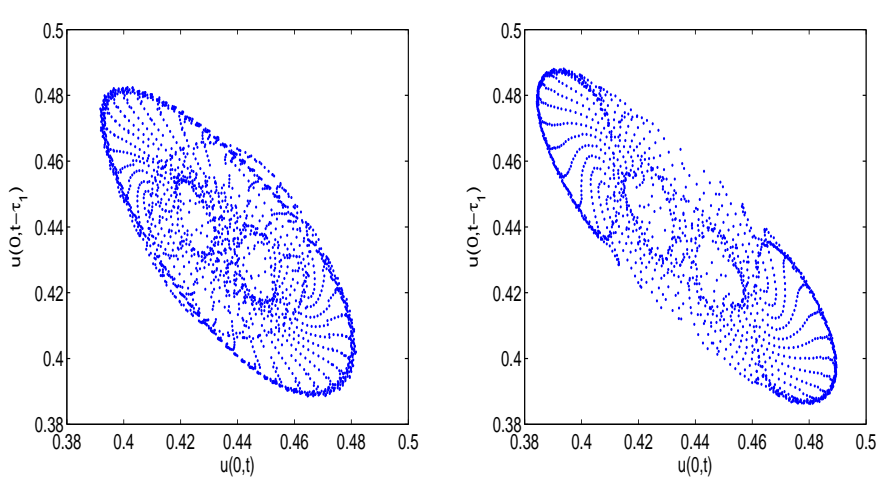

FIG. 12. The phase portraits in $u(0, t)-v(0, t)-u\left(0, t-\tau_{1}\right)$, and the corresponding Poincaré map on a Poincaré section $v(0, t)=v^{*}$ and $\dot{u}(x, t)=0$. The parameter is given as a) $\tau_{1}=3.82, \tau_{2}=1.4345$ in $D_{4}$, b) $\tau_{1}=3.9043, \tau_{2}=1.418$ near $L_{4}$, c) $\tau_{1}=3.905, \tau_{2}=1.4136$ in region $D_{6}$, respectively. Note that the transient states have been deleted for a clear expression.

been proposed for a long time. However, the theorem with two parameters has not been well stated. In this paper, we define Hopf bifurcation curve on the plane $\left(\tau_{1}, \tau_{2}\right)$, and give the sufficient condition of the existence of Hopf bifurcation in two-parameter plane.

The derivation process of normal form for double Hopf bifurcation is very difficult, and the calculation is very long. It is even harder when we deal with systems with two simultaneously 
varying delays, since the change of time scale $t \rightarrow \frac{t}{\tau_{1}}$ only transforms one delay to 1 , and the other delay becomes $\frac{\tau_{2}}{\tau_{1}}$, which makes the Taylor expansion of the nonlinear term $G\left(\sigma, U^{t}\right)$ with $U^{t}\left(-\frac{\tau_{2}}{\tau_{1}}\right)=U^{t}\left(-\frac{\tau_{2}^{*}+\sigma_{2}}{\tau_{1}^{*}+\sigma_{1}}\right)$ very complicated. We should notice that the method of calculation of normal form used here can be also used in other systems with two delays, one delay or without delay by slight modifications. The calculation formula of the normal form we give here is at a double Hopf bifurcation point with $k_{1}=k_{2}=0$. The cases of $k_{1}=0, k_{2} \neq 0$ and $k_{1} \neq 0, k_{2} \neq 0$ can be deduced in a similar way, but in our model these double Hopf bifurcation points are not located at the boundary of stability region, hence unstable manifold always exists.

\section{SUPPLEMENTARY MATERIAL}

In the supplementary material, we give the detailed calculation process of second and third order normal forms near double Hopf bifurcation.

\section{ACKNOWLEDGMENTS}

The authors are grateful to the handling editor and anonymous referees for their careful reading of the manuscript and valuable comments, which improve the exposition of the paper very much. Y. Du is supported by Education Department of Shaanxi Province (grant No. 18JK0123). B. Niu and J. Wei are supported by National Natural Science Foundation of China (grant Nos.11701120 and No.11771109) and the Foundation for Innovation at $\operatorname{HIT}(\mathrm{WH})$.

[1] P. Leslie, "A stochastic model for studying the properties of certain biological systems by numerical methods," Biometrika 45, 16-31 (1958).

[2] P. Leslie and J. Gower, "The properties of a stochastic model for the predator-prey type of interaction between two species," Biometrika 47, 219-234 (1960).

[3] M. Aziz, "Study of a Leslie-Gower-type tritrophic population," Chaos Soliton Fract. 14, 1275$1293(2002)$. 
[4] J. Collings, "The effects of the functional response on the bifurcation behavior of a mite predator-prey interaction model," J. Math. Biol. 36, 149-168 (1997).

[5] P. Feng and Y. Kang, "Dynamics of a modified Leslie-Gower model with double Allee efects," Nonlinear Dynam. 80, 1051-1062 (2015).

[6] Y. Ma, "Global Hopf bifurcation in the Leslie-Gower predator-prey model with two delays," Nonl. Anal. Real World Appl. 13, 370-375 (2012).

[7] J. Zhou, "Positive steady state solutions of a Leslie-Gower predator-prey model with Holling type II functional response and density-dependent diffusion," Nonlinear Anal. Theor. 82, 47-65 (2013).

[8] S. Yuan and Y. Song, "Stability and Hopf bifurcations in a delayed Leslie-Gower predator-prey system," J. Math. Anal. Appl. 355, 82-100 (2009).

[9] S. Yuan and Y. Song, "Bifurcation and stability analysis for a delayed Leslie-Gower predatorprey system," IMA J. Appl. Math. 74, 574-603 (2009).

[10] F. Chen, L. Chen, and X. Xie, "On a Leslie-Gower predator-prey model incorporating a prey refuge," Nonl. Anal. Real World Appl. 10, 2905-2908 (2009).

[11] R. M. May, "Time delay versus stability in population models with two and three trophic levels," Ecology 4, 315-325 (1973).

[12] R. Yang and J. Wei, "The effect of delay on a diffusive predator-prey system with modified Leslie-Gower functional response," B. Malays. Math. Sci. So. 40, 51-73 (2017).

[13] A. F. Nindjin, M. A. Aziz-Alaoui, and M. Cadivel, "Analysis of a predator-prey model with modified Leslie-Gower and Holling-type II schemes with time delay," Nonl. Anal. Real World Appl. 7, 1104-1118 (2006).

[14] Q. Liu, Y. Lin, and J. Cao, "Global Hopf bifurcation on two-delays Leslie-Gower predator-prey system with a prey refuge," Comput. Math. Method. M. 6, 619132 (2014).

[15] Y. Song, Y. Peng, and J. Wei, "Bifurcations for a predator-prey system with two delays," J. Math. Anal. Appl. 337, 466-479 (2008).

[16] K. Li and J. Wei, "Stability and Hopf bifurcation analysis of a prey-predator system with two delays," Chaos, Soliton. Fract. 42, 2606-2613 (2009).

[17] S. Ruan and J. Wei, "On the zeros of a third degree exponential polynomial with applications to a delayed model for the control of testosterone secretion," Ima J. Math. Appl. Med. 18, 41-52 (2001). 
[18] C. Xu, X. Tang, M. Liao, and X. He, Bifurcation analysis in a delayed Lokta-Volterra predatorprey model with two delays," Nonlinear Dynam. 66, 169-183 (2011).

[19] L. Deng, X. Wang, and M. Peng, "Hopf bifurcation analysis for a ratio-dependent predatorprey system with two delays and stage structure for the predator," Appl. Math. Comput. 231, 214-230 (2014).

[20] K. Gu, S. Niculescu, and J. Chen, "On stability crossing curves for general systems with two delays," J. Math. Anal. Appl. 311, 231-253 (2005).

[21] X. Lin and H. Wang, "Stability analysis of delay differential equations with two discrete delays," Can. Appl. Math. Q. 20, 519-533 (2012).

[22] Y. Du and S. B. Hsu, "A diffusive predator-prey model in heterogeneous environment," J. Differ. Equations 203, 331-364 (2004).

[23] S. Chen, J. Shi, and J. Wei, "Global stability and Hopf bifurcation in a delayed diffusive Leslie-Gower predator-prey system," Int. J. Bifurcat. Chaos 22, 331-517 (2012).

[24] I. Al-Darabsah, X. Tang, and Y. Yuan, "A prey-predator model with migrations and delays," Discrete Contin. Dyn. Syst. Ser. B 21, 737-761 (2016).

[25] C. Tian, "Delay-driven spatial patterns in a plankton allelopathic system," Chaos 22, 013129 (2012) .

[26] R. Yang and C. Zhang, "Dynamics in a diffusive modified Leslie-Gower predator-prey model with time delay and prey harvesting," Nonlinear Dynam. 87, 863-878 (2017).

[27] T. Faria, "Normal forms and Hopf bifurcation for partial differential equations with delays," Trans. Amer. Math. Soc. 352, 2217-2238 (2000).

[28] T. Faria and L. T. Magalhães, "Normal forms for retarded functional differential equations with parameters and applications to Hopf bifurcation," J. Differ. Equations 122, 181-200 (1995).

[29] K. L. Cooke and P. van den Driessche, "Analysis of an SEIRS epidemic model with two delays," J. Math. Biol. 35, 240-260 (1996).

[30] M. Jackson and B. M. Chen-Charpentier, "Modeling plant virus propagation with delays," J. Comput. Appl. Math. 309, 611-621 (2016).

[31] H. I. Freedman and V. S. H. Rao, "Stability criteria for a system involving two time delays," SIAM J. Appl. Math. 46, 552-560 (1986). 
[32] J. Wei and S. Ruan, "Stability and bifurcation in a neural network model with two delays," Physica D 130, 255-272 (1999).

[33] R. M. Nguimdo, "Constructing Hopf bifurcation lines for the stability of nonlinear systems with two time delays," Phys. Rev. E 97, 032211 (2018).

[34] Y. A. Kuznetsov, Elements of Applied Bifurcation Theory (Springer, 2013).

[35] J. Guckenheimer and P. Holmes, Nonlinear Oscillations, Dynamical Systems, and Bifurcations of Vector Fields (Springer, 1983).

[36] J. Hale, Theory of Functional Differential Equations (Springer-Verlag, 1977).

[37] K. L. Cooke and Z. Grossman, "Discrete delay, distributed delay and stability switches," J. Math. Anal. Appl. 86, 592-627 (1982).

[38] J. Wu, Theory and Applications of Partial Functional-Differential Equations (Springer, 1996).

[39] P. Battelino, C. Grebogi, E. Ott and J. Yorke, "Chaotic attractors on a 3-torus, and torus break-up," Physica D 39, 299-314 (1989).

[40] D. Ruelle and F. Takens, "On the nature of turbulence," Comm. Math. Phys. 20, 167-192 (1971).

[41] J. Eckmann, "Roads to turbulence in dissipative dynamical systems," Rev. Modern Phys. 53, 643-654 (1981). 